\title{
ERROR BOUNDS FOR SMALL JUMPS OF LÉVY PROCESSES
}

\author{
E. H. A. DIA, ${ }^{*}$ Université Paris-Est
}

\begin{abstract}
The pricing of options in exponential Lévy models amounts to the computation of expectations of functionals of Lévy processes. In many situations, Monte Carlo methods are used. However, the simulation of a Lévy process with infinite Lévy measure generally requires either truncating or replacing the small jumps by a Brownian motion with the same variance. We will derive bounds for the errors generated by these two types of approximation.
\end{abstract}

Keywords: Approximation of small jumps; Lévy process; Skorokhod embedding; Spitzer identity

2010 Mathematics Subject Classification: Primary 60G51; 65N15

Secondary $60 \mathrm{~J} 75$

\section{Introduction}

In recent years, the use of general Lévy processes in financial models has grown extensively (see [2], [5], and [11]). A variety of numerical methods have been subsequently developed, in particular methods based on Fourier analysis (see [4], [12], [13], and [15]). Nonetheless, in many situations, Monte Carlo methods have to be used. The simulation of a Lévy process with infinite Lévy measure is not straightforward, except in some special cases like the gamma or inverse Gaussian models. In practice, the small jumps of the Lévy process are either just truncated or replaced by a Brownian motion with the same variance (see [1], [7], [8], [16], and [18]). The latter approach was introduced by Asmussen and Rosinski [1], who showed that, under suitable conditions, the normalized cumulated small jumps asymptotically behave like a Brownian motion.

The purpose of this paper is to derive bounds for the errors generated by these two methods of approximation in the computation of functions of Lévy processes at a fixed time or functionals of the whole path of Lévy processes. We also derive bounds for the cumulative distribution functions. These bounds can be used to determine which type of approximations to use, since replacing small jumps by Brownian motion is more time consuming (if we use Monte Carlo methods). Our bounds can be applied to derive approximation errors for lookback, barrier, American, or Asian options. But, this latter point will not be developed, and is left to another paper.

The characteristic function of a real Lévy process $X$ with generating triplet $\left(\gamma, b^{2}, v\right)$ is given by

$$
\mathbb{E} \mathrm{e}^{\mathrm{i} u X_{t}}=\exp \left\{t\left(\mathrm{i} \gamma u-\frac{b^{2} u^{2}}{2}+\int_{-\infty}^{+\infty}\left(\mathrm{e}^{\mathrm{i} u x}-1-\mathrm{i} u x \mathbf{1}_{\{|x| \leq 1\}}\right) \nu(\mathrm{d} x)\right)\right\},
$$

Received 17 February 2011; revision received 19 November 2012.

* Postal address: Laboratoire d'Analyse et de Mathématiques Appliquées, Université Paris-Est, UMR CNRS 8050, 5 boulevard Descartes, Champs-sur-Marne, 77454 Marne-la-Vallée, France. Email address: dia.eha@gmail.com 
where $\gamma \in \mathbb{R}, b \geq 0$, and $v$ is a Lévy measure. The process $X$ is the independent sum of a drift term $\gamma t$, a Brownian component $b B_{t}$, and a compensated jump part with Lévy measure $v$. The process $X$ has finite or infinite activity if $v(\mathbb{R})<\infty$ or, respectively, $v(\mathbb{R})=+\infty$.

For $0<\varepsilon \leq 1$, the process $X^{\varepsilon}$ is defined by

$$
X_{t}^{\varepsilon}=\gamma t+b B_{t}+\sum_{0 \leq s \leq t} \Delta X_{s} \mathbf{1}_{\left\{\left|\Delta X_{s}\right|>\varepsilon\right\}}-t \int_{\varepsilon<|x| \leq 1} x v(\mathrm{~d} x) .
$$

The process $X^{\varepsilon}$ is obtained (from $X$ ) by subtracting the compensated sum of jumps not exceeding $\varepsilon$ in absolute value. Let

$$
R^{\varepsilon}=X-X^{\varepsilon}
$$

The process $R^{\varepsilon}$ is a Lévy process with characteristic function

$$
\mathbb{E} \mathrm{e}^{\mathrm{i} u R_{t}^{\varepsilon}}=\exp \left\{t \int_{|x| \leq \varepsilon}\left(\mathrm{e}^{\mathrm{i} u x}-1-\mathrm{i} u x\right) v(\mathrm{~d} x)\right\} .
$$

It holds that $\mathbb{E}\left(R_{t}^{\varepsilon}\right)=0$ and $\operatorname{var}\left(R_{t}^{\varepsilon}\right)=\sigma(\varepsilon)^{2} t$, where

$$
\sigma(\varepsilon)=\sqrt{\int_{|x| \leq \varepsilon} x^{2} v(\mathrm{~d} x)}
$$

Note that $\lim _{\varepsilon \rightarrow 0} \sigma(\varepsilon)=0$. The behavior of $\sigma(\varepsilon)$ when $\varepsilon$ goes to 0 is known for classical models (VG, NIG, CGMY, etc.). As noted in Example 2.3 of [1], if $v(\mathrm{~d} x)=|x|^{-1-\alpha} L(x) \mathrm{d} x$, where $\alpha \in$ $(0,2)$ and $L$ is slowly varying at 0 , then it holds that $\sigma(\varepsilon) \sim((L(-\varepsilon)+L(\varepsilon)) /(2-\alpha))^{1 / 2} \varepsilon^{1-\alpha / 2}$; consequently, $\lim _{\varepsilon \rightarrow 0} \sigma(\varepsilon) / \varepsilon=+\infty$.

We also define the process $\hat{X}^{\varepsilon}$ by

$$
\hat{X}_{t}^{\varepsilon}=X_{t}^{\varepsilon}+\sigma(\varepsilon) \hat{W}_{t}, \quad t \geq 0,
$$

where $\hat{W}$ is a standard Brownian motion independent of $X$. We aim to study the behavior of the errors made by replacing $X$ by $X^{\varepsilon}$ or $\hat{X}^{\varepsilon}$, with respect to the level $\varepsilon$. These errors are studied for the process $X$ at a fixed date and for its running supremum. Set, for any $t \geq 0$,

$$
M_{t}=\sup _{0 \leq s \leq t} X_{s}, \quad M_{t}^{\varepsilon}=\sup _{0 \leq s \leq t} X_{s}^{\varepsilon}, \quad \hat{M}_{t}^{\varepsilon}=\sup _{0 \leq s \leq t} \hat{X}_{s}^{\varepsilon} .
$$

Unless stated otherwise, $X$ is a Lévy process with generating triplet $\left(\gamma, b^{2}, v\right)$.

The paper is organized as follows. In the next section we will study the errors resulting from the truncation of the compensated sum of small jumps. The results of that section are based on estimates for the moments of $R^{\varepsilon}$. We also derive an estimate for the expectation $\mathbb{E}\left(M_{t}-M_{t}^{\varepsilon}\right)$, by using Spitzer's identity. In Section 3 we study the errors resulting from a Brownian approximation. The process $X$ will be approximated by the process $\hat{X}^{\varepsilon}$. A major result of Section 3 is Theorem 2, which gives an error bound for the expectation of a function of the supremum. This result is the consequence of Theorem 3, which relies on the Skorokhod embedding theorem. 


\section{Truncation of the compensated sum of small jumps}

In this section we will study the errors resulting from the approximation of $X$ by $X^{\varepsilon}$. These errors are related to the moments of $R^{\varepsilon}$. Define

$$
\sigma_{0}(\varepsilon)=\max (\sigma(\varepsilon), \varepsilon) .
$$

The next result will be useful for many proofs in this paper.

Proposition 1. Let $X$ be a Lévy process, and let $R^{\varepsilon}$ be as defined in (1). Then

$$
\mathbb{E}\left|R_{t}^{\varepsilon}\right|^{4}=t \int_{|x| \leq \varepsilon} x^{4} v(\mathrm{~d} x)+3\left(t \sigma(\varepsilon)^{2}\right)^{2},
$$

and, for any real $q>0$,

$$
\mathbb{E}\left|R_{t}^{\varepsilon}\right|^{q} \leq K_{q, t} \sigma_{0}(\varepsilon)^{q},
$$

where $K_{q, t}$ is a positive constant which depends only on $q$ and $t$.

Proof. Let $c_{k}\left(R_{t}^{\varepsilon}\right)$ denote the $k$ th cumulant of $R_{t}^{\varepsilon}$. Then $c_{1}\left(R_{t}^{\varepsilon}\right)=\mathbb{E}\left(R_{t}^{\varepsilon}\right)=0$, and, for any $k \geq 2, c_{k}\left(R_{t}^{\varepsilon}\right)=t \int_{|x| \leq \varepsilon} x^{k} v(\mathrm{~d} x)$ (note that $\left.c_{2}\left(R_{t}^{\varepsilon}\right)=\operatorname{var}\left(R_{t}^{\varepsilon}\right)=\sigma^{2}(\varepsilon) t\right)$. See Proposition 1.2 of [20]. Substituting into the general formula

$$
\mu_{4}^{\prime}=c_{4}+4 c_{3} c_{1}+3 c_{2}^{2}+6 c_{2} c_{1}^{2}+c_{1}^{4}
$$

(cf. (4) below), where, here and below, $\mu_{k}^{\prime}$ and $c_{k}$ denote the $k$ th moment and $k$ th cumulant of a distribution, respectively, gives the first part of the proposition. We now prove the second part. Let $n=\lceil q / 2\rceil$. Since $0<q /(2 n) \leq 1$,

$$
\mathbb{E}\left|R_{t}^{\varepsilon}\right|^{q} \leq\left(\mathbb{E}\left|R_{t}^{\varepsilon}\right|^{2 n}\right)^{q /(2 n)}
$$

(by Jensen's inequality for concave functions). It thus suffices to prove the result for the case $q=2 n, n \in \mathbb{N}$; in fact, for any $n \in \mathbb{N}$, it holds that

$$
\left|\mathbb{E}\left(R_{t}^{\varepsilon}\right)^{n}\right| \leq K_{n, t} \sigma_{0}(\varepsilon)^{n} .
$$

The last inequality can be proved by induction as follows. It is trivial for $n=0,1,2$. Suppose that (3) holds for all $n<m$. Then, by the well-known result (see, e.g. Theorem 2 of [14])

$$
\mu_{m}^{\prime}=\sum_{n=0}^{m-1}\left(\begin{array}{c}
m-1 \\
n
\end{array}\right) \mu_{n}^{\prime} c_{m-n}, \quad m \geq 1
$$

for all $m \geq 2$, we have (recall that $c_{1}\left(R_{t}^{\varepsilon}\right)=0$ )

$$
\left|\mathbb{E}\left(R_{t}^{\varepsilon}\right)^{m}\right| \leq \sum_{n=0}^{m-2}\left(\begin{array}{c}
m-1 \\
n
\end{array}\right)\left|\mathbb{E}\left(R_{t}^{\varepsilon}\right)^{n}\right|\left|c_{m-n}\left(R_{t}^{\varepsilon}\right)\right| .
$$

Hence, in view of the induction hypothesis, it suffices to show that $\left|c_{m-n}\left(R_{t}^{\varepsilon}\right)\right| \leq t \sigma_{0}(\varepsilon)^{m-n}$. Since $m-n \geq 2$, we have $c_{m-n}\left(R_{t}^{\varepsilon}\right)=t \int_{|x| \leq \varepsilon} x^{m-n} v(\mathrm{~d} x)$, and, hence,

$$
\left|c_{m-n}\left(R_{t}^{\varepsilon}\right)\right| \leq t \int_{|x| \leq \varepsilon}|x|^{m-n} \nu(\mathrm{d} x) \leq t \varepsilon^{m-n-2} \int_{|x| \leq \varepsilon}|x|^{2} \nu(\mathrm{d} x) \leq t \sigma_{0}(\varepsilon)^{m-n} .
$$

The proposition is thus established. 


\subsection{Estimates for smooth functions}

Let $X$ be a Lévy process, and let $f$ be a $C$-Lipschitz function, where $C>0$. Then

$$
\mathbb{E}\left|f\left(X_{t}\right)-f\left(X_{t}^{\varepsilon}\right)\right| \leq C \mathbb{E}\left|R_{t}^{\varepsilon}\right| \leq C \sqrt{\mathbb{E}\left|R_{t}^{\varepsilon}\right|^{2}} \leq C \sqrt{t} \sigma(\varepsilon) .
$$

Note that we do not ask that $f\left(X_{t}\right)$ be integrable. If $f$ is more regular, sharper estimates can be derived, as shown in the following proposition.

Proposition 2. Let $X$ be an infinite activity Lévy process.

1. If $f \in C^{1}(\mathbb{R})$ and satisfies $\mathbb{E}\left|f^{\prime}\left(X_{t}^{\varepsilon}\right)\right|<\infty$, and if there exists $\beta>1$ such that $\left(\sup _{\varepsilon \in(0,1]} \mathbb{E}\left|f^{\prime}\left(X_{t}^{\varepsilon}+\theta R_{t}^{\varepsilon}\right)-f^{\prime}\left(X_{t}^{\varepsilon}\right)\right|^{\beta}\right)^{1 / \beta}$ is finite and integrable with respect to $\theta$ on $[0,1]$, then

$$
\mathbb{E}\left(f\left(X_{t}\right)-f\left(X_{t}^{\varepsilon}\right)\right)=o\left(\sigma_{0}(\varepsilon)\right) .
$$

2. If $f \in C^{2}(\mathbb{R})$ and satisfies $\mathbb{E}\left|f^{\prime}\left(X_{t}^{\varepsilon}\right)\right|+\mathbb{E}\left|f^{\prime \prime}\left(X_{t}^{\varepsilon}\right)\right|<\infty$, and if there exists $\beta>1$ such that $\left(\sup _{\varepsilon \in(0,1]} \mathbb{E}\left|f^{\prime \prime}\left(X_{t}^{\varepsilon}+\theta R_{t}^{\varepsilon}\right)-f^{\prime \prime}\left(X_{t}^{\varepsilon}\right)\right|^{\beta}\right)^{1 / \beta}$ is finite and integrable with respect to $\theta$ on $[0,1]$, then

$$
\mathbb{E}\left(f\left(X_{t}\right)-f\left(X_{t}^{\varepsilon}\right)\right)=\frac{\sigma(\varepsilon)^{2} t}{2} \mathbb{E} f^{\prime \prime}\left(X_{t}^{\varepsilon}\right)+o\left(\sigma_{0}(\varepsilon)^{2}\right) .
$$

Note that, if $f$ has bounded derivatives or $f$ is the exponential function and $\mathrm{e}^{\beta X_{t}}$ is integrable, where $\beta>1$, the conditions in the above proposition are satisfied. Recall that the truncation of small jumps is used when $v(\mathbb{R})=\infty$. In typical applications, we have $\lim \inf \sigma(\varepsilon) / \varepsilon>0$, so that $o\left(\sigma_{0}(\varepsilon)^{2}\right)$ is in fact $o\left(\sigma(\varepsilon)^{2}\right)$.

Proof of Proposition 2. To prove part 1, we first write $f\left(X_{t}\right)-f\left(X_{t}^{\varepsilon}\right)$ as

$$
f\left(X_{t}\right)-f\left(X_{t}^{\varepsilon}\right)=\int_{0}^{1}\left(f^{\prime}\left(X_{t}^{\varepsilon}+\theta R_{t}^{\varepsilon}\right)-f^{\prime}\left(X_{t}^{\varepsilon}\right)\right) R_{t}^{\varepsilon} \mathrm{d} \theta+f^{\prime}\left(X_{t}^{\varepsilon}\right) R_{t}^{\varepsilon}
$$

(by Theorem 27.4 of [17], $R_{t}^{\varepsilon} \neq 0$ almost surely (a.s.)). Since $R_{t}^{\varepsilon}$ and $X_{t}^{\varepsilon}$ are independent, $\mathbb{E}\left[f^{\prime}\left(X_{t}^{\varepsilon}\right) R_{t}^{\varepsilon}\right]=0$. For any $1<\alpha<\beta$, by Hölder's inequality,

$$
\mathbb{E}\left|\left(f^{\prime}\left(X_{t}^{\varepsilon}+\theta R_{t}^{\varepsilon}\right)-f^{\prime}\left(X_{t}^{\varepsilon}\right)\right) R_{t}^{\varepsilon}\right| \leq\left(\mathbb{E}\left|f^{\prime}\left(X_{t}^{\varepsilon}+\theta R_{t}^{\varepsilon}\right)-f^{\prime}\left(X_{t}^{\varepsilon}\right)\right|^{\alpha}\right)^{1 / \alpha}\left(\mathbb{E}\left|R_{t}^{\varepsilon}\right|^{\alpha /(\alpha-1)}\right)^{(\alpha-1) / \alpha} .
$$

By Lyapunov's inequality,

$$
\left(\mathbb{E}\left|f^{\prime}\left(X_{t}^{\varepsilon}+\theta R_{t}^{\varepsilon}\right)-f^{\prime}\left(X_{t}^{\varepsilon}\right)\right|^{\alpha}\right)^{1 / \alpha} \leq\left(\mathbb{E}\left|f^{\prime}\left(X_{t}^{\varepsilon}+\theta R_{t}^{\varepsilon}\right)-f^{\prime}\left(X_{t}^{\varepsilon}\right)\right|^{\beta}\right)^{1 / \beta} .
$$

Furthermore, the assumption $\sup _{\varepsilon \in(0,1]} \mathbb{E}\left|f^{\prime}\left(X_{t}^{\varepsilon}+\theta R_{t}^{\varepsilon}\right)-f^{\prime}\left(X_{t}^{\varepsilon}\right)\right|^{\beta}<\infty$ implies that the collection $\left\{\left|f^{\prime}\left(X_{t}^{\varepsilon}+\theta R_{t}^{\varepsilon}\right)-f^{\prime}\left(X_{t}^{\varepsilon}\right)\right|^{\alpha}\right\}_{\varepsilon \in(0,1]}$ is uniformly integrable; hence, since $\mid f^{\prime}\left(X_{t}^{\varepsilon}+\right.$ $\left.\theta R_{t}^{\varepsilon}\right)-\left.f^{\prime}\left(X_{t}^{\varepsilon}\right)\right|^{\alpha} \rightarrow 0$ a.s. as $\varepsilon \rightarrow 0, \mathbb{E}\left|f^{\prime}\left(X_{t}^{\varepsilon}+\theta R_{t}^{\varepsilon}\right)-f^{\prime}\left(X_{t}^{\varepsilon}\right)\right|^{\alpha} \rightarrow 0$ (pointwise for $\theta \in$ $[0,1])$. Therefore, by dominated convergence,

$$
\lim _{\varepsilon \rightarrow 0} \int_{0}^{1}\left(\mathbb{E}\left|f^{\prime}\left(X_{t}^{\varepsilon}+\theta R_{t}^{\varepsilon}\right)-f^{\prime}\left(X_{t}^{\varepsilon}\right)\right|^{\alpha}\right)^{1 / \alpha} \mathrm{d} \theta=0 .
$$

Combined with Proposition 1, it thus follows that

$$
\int_{0}^{1} \mathbb{E}\left[\left(f^{\prime}\left(X_{t}^{\varepsilon}+\theta R_{t}^{\varepsilon}\right)-f^{\prime}\left(X_{t}^{\varepsilon}\right)\right) R_{t}^{\varepsilon}\right] \mathrm{d} \theta=o\left(\sigma_{0}(\varepsilon)\right) .
$$


Part 1 of the proposition then follows from (5) (using Fubini's theorem). We now prove the second part of the proposition. Using Taylor's formula, we obtain

$$
\begin{aligned}
\mathbb{E}\left(f\left(X_{t}\right)-f\left(X_{t}^{\varepsilon}\right)\right)= & \mathbb{E}\left[f^{\prime}\left(X_{t}^{\varepsilon}\right)\left(X_{t}-X_{t}^{\varepsilon}\right)+\int_{X_{t}^{\varepsilon}}^{X_{t}} f^{\prime \prime}(x)\left(X_{t}-x\right) \mathrm{d} x\right] \\
= & \mathbb{E}\left[f^{\prime}\left(X_{t}^{\varepsilon}\right) R_{t}^{\varepsilon}+\int_{0}^{1} f^{\prime \prime}\left(X_{t}^{\varepsilon}+\theta R_{t}^{\varepsilon}\right)(1-\theta)\left(R_{t}^{\varepsilon}\right)^{2} \mathrm{~d} \theta\right] \\
= & \mathbb{E}\left[\int_{0}^{1} f^{\prime \prime}\left(X_{t}^{\varepsilon}+\theta R_{t}^{\varepsilon}\right)(1-\theta)\left(R_{t}^{\varepsilon}\right)^{2} \mathrm{~d} \theta\right] \\
= & \mathbb{E}\left[\int_{0}^{1} f^{\prime \prime}\left(X_{t}^{\varepsilon}\right)(1-\theta)\left(R_{t}^{\varepsilon}\right)^{2} \mathrm{~d} \theta\right] \\
& +\mathbb{E}\left[\int_{0}^{1}\left(f^{\prime \prime}\left(X_{t}^{\varepsilon}+\theta R_{t}^{\varepsilon}\right)-f^{\prime \prime}\left(X_{t}^{\varepsilon}\right)\right)(1-\theta)\left(R_{t}^{\varepsilon}\right)^{2} \mathrm{~d} \theta\right] .
\end{aligned}
$$

The first expectation after the last equality sign is equal to $\left(\sigma(\varepsilon)^{2} t / 2\right) \mathbb{E} f^{\prime \prime}\left(X_{t}^{\varepsilon}\right)$, while the second expectation can be shown to be $o\left(\sigma_{0}(\varepsilon)^{2}\right)$ by following the proof of part 1 . The proposition is proved.

Remark 1. Assume that $X$ is an integrable infinite activity Lévy process and that $f \in C^{1}(\mathbb{R})$ with $f^{\prime}$ being $C$-Lipschitz. Then

$$
\left|\mathbb{E}\left(f\left(X_{t}\right)-f\left(X_{t}^{\varepsilon}\right)\right)\right| \leq \frac{C \sigma(\varepsilon)^{2} t}{2} .
$$

Indeed, $\mathbb{E}\left[f^{\prime}\left(X_{t}^{\varepsilon}\right) R_{t}^{\varepsilon}\right]=0$ (by the assumptions on $X$ and $f, \mathbb{E}\left|f^{\prime}\left(X_{t}^{\varepsilon}\right)\right|<\infty$ ), and so the result follows directly from (5) using

$$
\left|\mathbb{E}\left(f\left(X_{t}\right)-f\left(X_{t}^{\varepsilon}\right)\right)\right| \leq \mathbb{E}\left[\int_{0}^{1}\left|f^{\prime}\left(X_{t}^{\varepsilon}+\theta R_{t}^{\varepsilon}\right)-f^{\prime}\left(X_{t}^{\varepsilon}\right)\right|\left|R_{t}^{\varepsilon}\right| \mathrm{d} \theta\right] .
$$

We will consider now the case of the supremum process.

Proposition 3. Let $X$ be a Lévy process, and let $f$ be a $K$-Lipschitz function. Then

$$
\mathbb{E}\left|f\left(M_{t}\right)-f\left(M_{t}^{\varepsilon}\right)\right| \leq 2 K \sqrt{t} \sigma(\varepsilon) .
$$

Proof. We have

$$
\begin{aligned}
\mathbb{E}\left|f\left(\sup _{0 \leq s \leq t} X_{s}\right)-f\left(\sup _{0 \leq s \leq t} X_{s}^{\varepsilon}\right)\right| & \leq K \mathbb{E}\left|\sup _{0 \leq s \leq t} X_{s}-\sup _{0 \leq s \leq t} X_{s}^{\varepsilon}\right| \\
& \leq K \mathbb{E} \sup _{0 \leq s \leq t}\left|R_{s}^{\varepsilon}\right| \\
& \leq K \sqrt{\mathbb{E}\left(\sup _{0 \leq s \leq t}\left|R_{s}^{\varepsilon}\right|\right)^{2}} .
\end{aligned}
$$

Note that $R^{\varepsilon}$ is a càdlàg martingale. So, using Doob's inequality, we obtain

$$
\mathbb{E}\left|f\left(\sup _{0 \leq s \leq t} X_{s}\right)-f\left(\sup _{0 \leq s \leq t} X_{s}^{\varepsilon}\right)\right| \leq 2 K \sqrt{\mathbb{E}\left|R_{t}^{\varepsilon}\right|^{2}}=2 K \sqrt{t} \sigma(\varepsilon) .
$$


Remark 2. Suppose that $X$ is an integrable Lévy process and that $f$ is a function from $\mathbb{R}^{+} \times \mathbb{R}$ to $\mathbb{R}, K$-Lipschitz with respect to its second variable. Then

$$
\left|\sup _{\tau \in \mathcal{T}_{[0, t]}} \mathbb{E} f\left(\tau, X_{\tau}\right)-\sup _{\tau \in \mathcal{T}_{[0, t]}} \mathbb{E} f\left(\tau, X_{\tau}^{\varepsilon}\right)\right| \leq 2 K \sqrt{t} \sigma(\varepsilon),
$$

where $\mathcal{T}_{[0, t]}$ denotes the set of stopping times with values in $[0, t]$. For a proof, the reader is referred to [9, pp. 67-68].

The bound in Proposition 3 might not be optimal. This is what suggests the following result.

Theorem 1. Let $X$ be an integrable infinite activity Lévy process. Then

$$
0 \leq \mathbb{E}\left(M_{t}-M_{t}^{\varepsilon}\right)=o(\sigma(\varepsilon)) .
$$

Proof. Using Spitzer's identity (see Proposition 1 of [10, Section 3] for details), we have

$$
\mathbb{E}\left(M_{t}-M_{t}^{\varepsilon}\right)=\int_{0}^{t} \frac{\mathbb{E} X_{s}^{+}}{s} \mathrm{~d} s-\int_{0}^{t} \frac{\mathbb{E}\left(X_{s}^{\varepsilon}\right)^{+}}{s} \mathrm{~d} s=\int_{0}^{t} \mathbb{E}\left(X_{s}^{+}-\left(X_{s}^{\varepsilon}\right)^{+}\right) \frac{\mathrm{d} s}{s} .
$$

It holds that

$$
\begin{aligned}
X_{s}^{+}-\left(X_{s}^{\varepsilon}\right)^{+} & =\left(X_{s}^{\varepsilon}+R_{s}^{\varepsilon}\right)^{+}-\left(X_{s}^{\varepsilon}\right)^{+} \\
& =\left(X_{s}^{\varepsilon}+R_{s}^{\varepsilon}\right) \mathbf{1}_{\left\{X_{s}^{\varepsilon}+R_{s}^{\varepsilon}>0\right\}}-X_{s}^{\varepsilon} \mathbf{1}_{\left\{X_{s}^{\varepsilon}>0\right\}} \\
& =\left(X_{s}^{\varepsilon}+R_{s}^{\varepsilon}\right)\left(\mathbf{1}_{\left\{X_{s}^{\varepsilon}>0\right\}}+\mathbf{1}_{\left\{X_{s}^{\varepsilon}+R_{s}^{\varepsilon}>0, X_{s}^{\varepsilon} \leq 0\right\}}-\mathbf{1}_{\left\{X_{s}^{\varepsilon}+R_{s}^{\varepsilon} \leq 0, X_{s}^{\varepsilon}>0\right\}}\right)-X_{s}^{\varepsilon} \mathbf{1}_{\left\{X_{s}^{\varepsilon}>0\right\}} \\
& =\left(X_{s}^{\varepsilon}+R_{s}^{\varepsilon}\right)\left(\mathbf{1}_{\left\{X_{s}^{\varepsilon}+R_{s}^{\varepsilon}>0, X_{s}^{\varepsilon} \leq 0\right\}}-\mathbf{1}_{\left\{X_{s}^{\varepsilon}+R_{s}^{\varepsilon} \leq 0, X_{s}^{\varepsilon}>0\right\}}\right)+R_{s}^{\varepsilon} \mathbf{1}_{\left\{X_{s}^{\varepsilon}>0\right\}} \\
& =\left(\left|R_{s}^{\varepsilon}\right|-\left|X_{s}^{\varepsilon}\right|\right)^{+}\left(\mathbf{1}_{\left\{X_{s}^{\varepsilon}+R_{s}^{\varepsilon}>0, X_{s}^{\varepsilon} \leq 0\right\}}+\mathbf{1}_{\left\{X_{s}^{\varepsilon}+R_{s}^{\varepsilon} \leq 0, X_{s}^{\varepsilon}>0\right\}}\right)+R_{s}^{\varepsilon} \mathbf{1}_{\left\{X_{s}^{\varepsilon}>0\right\}} .
\end{aligned}
$$

Set $I_{s}^{\varepsilon}=\mathbb{E}\left(X_{s}^{+}-\left(X_{s}^{\varepsilon}\right)^{+}\right)$. Thus, since $\mathbb{E}\left(R_{s}^{\varepsilon} \mathbf{1}_{\left\{X_{s}^{\varepsilon}>0\right\}}\right)=0$ (by independence),

$$
0 \leq I_{s}^{\varepsilon} \leq \mathbb{E}\left(\left|R_{s}^{\varepsilon}\right|-\left|X_{s}^{\varepsilon}\right|\right)^{+} .
$$

By the left inequality, $\mathbb{E}\left(M_{t}-M_{t}^{\varepsilon}\right) \geq 0$. We now prove that $\mathbb{E}\left(M_{t}-M_{t}^{\varepsilon}\right)=o(\sigma(\varepsilon))$. Since $\left(\left|R_{s}^{\varepsilon}\right|-\left|X_{s}^{\varepsilon}\right|\right)^{+} \leq\left|R_{s}^{\varepsilon}\right| \mathbf{1}_{\left\{\left|X_{s}^{\varepsilon}\right|<\left|R_{s}^{\varepsilon}\right|\right\}}$, we obtain $I_{s}^{\varepsilon} \leq \mathbb{E}\left(\left|R_{s}^{\varepsilon}\right| \mathbf{1}_{\left\{\left|X_{s}^{\varepsilon}\right|<\left|R_{s}^{\varepsilon}\right|\right\}}\right)$. Hence, by the CauchySchwarz inequality,

$$
I_{s}^{\varepsilon} \leq\left(\mathbb{E}\left|R_{s}^{\varepsilon}\right|^{2}\right)^{1 / 2}\left(\mathbb{E}\left(\mathbf{1}_{\left\{\left|X_{s}^{\varepsilon}\right|<\left|R_{s}^{\varepsilon}\right|\right\}}\right)^{2}\right)^{1 / 2}=\sigma(\varepsilon) \sqrt{s} \mathbb{P}\left[\left|X_{s}^{\varepsilon}\right|<\left|R_{s}^{\varepsilon}\right|\right]^{1 / 2} .
$$

Thus,

$$
0 \leq \mathbb{E}\left(M_{t}-M_{t}^{\varepsilon}\right) \leq \sigma(\varepsilon) \int_{0}^{t} \mathbb{P}\left[\left|X_{s}^{\varepsilon}\right|<\left|R_{s}^{\varepsilon}\right|\right]^{1 / 2} \frac{\mathrm{d} s}{\sqrt{s}} .
$$

Since $v(\mathbb{R})=\infty, R_{s}^{\varepsilon} \rightarrow 0$ a.s. and $X_{s}^{\varepsilon} \rightarrow X_{s}$ a.s. with $X_{s} \neq 0$. Hence, $\mathbb{P}\left[\left|X_{s}^{\varepsilon}\right|<\left|R_{s}^{\varepsilon}\right|\right]^{1 / 2} \rightarrow 0$ as $\varepsilon \rightarrow 0$. Therefore, by dominated convergence,

$$
\lim _{\varepsilon \rightarrow 0} \int_{0}^{t} \mathbb{P}\left[\left|X_{s}^{\varepsilon}\right|<\left|R_{s}^{\varepsilon}\right|\right]^{1 / 2} \frac{\mathrm{d} s}{\sqrt{s}}=0,
$$

and so $\mathbb{E}\left(M_{t}-M_{t}^{\varepsilon}\right)=o(\sigma(\varepsilon))$.

In financial applications, the function $f$ in Proposition 3 is not always Lipschitz, as for the call lookback option where the function is exponential. 
Proposition 4. Let $X$ be a Lévy process, and let $p>1$. If $\mathbb{E}^{p M_{t}}<\infty$ then

$$
\mathbb{E}\left|\mathrm{e}^{M_{t}}-\mathrm{e}^{M_{t}^{\varepsilon}}\right| \leq C_{p, t} \sigma_{0}(\varepsilon),
$$

where $C_{p, t}$ is a positive constant independent of $\varepsilon$.

Lemma 1. Let $p>0$. If $\mathbb{E}^{p M_{t}}<\infty$ then $\sup _{0<\delta \leq 1} \mathbb{E}^{p M_{t}^{\delta}}<\infty$.

Remark 3. For any $p>0, \mathbb{E} \mathrm{e}^{p M_{t}}<\infty$ if and only if $\int_{x>1} \mathrm{e}^{p x} v(\mathrm{~d} x)<\infty$.

The 'only if' part follows from Theorem 25.3 of [17], noting that $\mathrm{e}^{p X_{t}} \leq \mathrm{e}^{p M_{t}}$. For the 'if' part, decompose $X$ as the independent sum $X=Y+Z+Z^{\prime}$ of Lévy processes, where $Y$ has Lévy measure $[v]_{\{|x| \leq 1\}}$, and $Z$ and $Z^{\prime}$ are pure jump with Lévy measures $[v]_{\{x>1\}}$ and $[v]_{\{x<-1\}}$, respectively. Here $[v]_{E}$ denotes the restriction of $v$ to $E$. Note that $M_{t} \leq \sup _{0 \leq s \leq t} Y_{s}+Z_{t}$; thus, $\mathbb{E}\left[\mathrm{e}^{p M_{t}}\right] \leq \mathbb{E}\left[\exp \left\{p \sup _{0 \leq s \leq t} Y_{s}\right\}\right] \mathbb{E}\left[\mathrm{e}^{p Z_{t}}\right]$. It can be deduced from Theorems 25.3 and 25.18 of [17] that $\mathbb{E}\left[\exp \left\{p \sup _{0 \leq s \leq t} Y_{s}\right\}\right]$ is finite; so is $\mathbb{E}\left[\mathrm{e}^{p Z_{t}}\right]$ by the former theorem, under the assumption that $\int_{x>1} \mathrm{e}^{p x} v(\mathrm{~d} x)<\infty$. Hence, $\mathbb{E}\left[\mathrm{e}^{p M_{t}}\right]<\infty$.

Proof of Lemma 1. For $\delta \in(0,1]$, define $\bar{R}^{\delta}=X^{\delta}-X^{1}$. The process $\bar{R}^{\delta}$ is the compensated sum of jumps belonging to $(\delta, 1]$ in absolute value. So

$$
\mathbb{E}^{p M_{t}^{\delta}} \leq \mathbb{E} \exp \left\{p \sup _{0 \leq s \leq t} X_{s}^{1}+p \sup _{0 \leq s \leq t} \bar{R}_{s}^{\delta}\right\} \leq \mathbb{E} \exp \left\{p \sup _{0 \leq s \leq t} X_{s}^{1}\right\} \mathbb{E} \exp \left\{p \sup _{0 \leq s \leq t}\left|\bar{R}_{s}^{\delta}\right|\right\} .
$$

By hypothesis and Remark 3, noting that Remark 3 also holds for $M_{t}^{1}, \mathbb{E} \exp \left\{p \sup _{0 \leq s \leq t} X_{s}^{1}\right\}<$ $\infty$. We need to bound $\mathbb{E} \exp \left\{p \sup _{0 \leq s \leq t}\left|\bar{R}_{s}^{\delta}\right|\right\}$ independently of $\delta$. We have

$$
\begin{aligned}
\mathbb{E} \exp \left\{p \sup _{0 \leq s \leq t}\left|\bar{R}_{s}^{\delta}\right|\right\} & =\mathbb{E} \sum_{n=0}^{+\infty} \frac{\left(p \sup _{0 \leq s \leq t}\left|\bar{R}_{s}^{\delta}\right|\right)^{n}}{n !} \\
& =1+p \mathbb{E} \sup _{0 \leq s \leq t}\left|\bar{R}_{s}^{\delta}\right|+\sum_{n=2}^{+\infty} \frac{p^{n}}{n !} \mathbb{E}\left(\sup _{0 \leq s \leq t}\left|\bar{R}_{s}^{\delta}\right|\right)^{n} .
\end{aligned}
$$

By Doob's inequality ( $\bar{R}^{\delta}$ is a càdlàg martingale),

$$
\begin{aligned}
\mathbb{E} \exp \left\{p \sup _{0 \leq s \leq t}\left|\bar{R}_{s}^{\delta}\right|\right\} & \leq 1+p \sqrt{\mathbb{E}\left(\sup _{0 \leq s \leq t}\left|\bar{R}_{s}^{\delta}\right|\right)^{2}}+\sum_{n=2}^{+\infty} \frac{p^{n}}{n !}\left(\frac{n}{n-1}\right)^{n} \mathbb{E}\left|\bar{R}_{t}^{\delta}\right|^{n} \\
& \leq 1+2 p \sqrt{\mathbb{E}\left|\bar{R}_{t}^{\delta}\right|^{2}}+\sum_{n=2}^{+\infty} \frac{p^{n}}{n !} 2^{n} \mathbb{E}\left|\bar{R}_{t}^{\delta}\right|^{n} \\
& \leq 2 p \sqrt{\operatorname{var}\left(\bar{R}_{t}^{\delta}\right)}+\mathbb{E} \sum_{n=0}^{+\infty} \frac{p^{n}}{n !} 2^{n}\left|\bar{R}_{t}^{\delta}\right|^{n} \\
& \leq 2 p \sqrt{t \int_{\delta<|x| \leq 1} x^{2} v(\mathrm{~d} x)}+\mathbb{E}^{2 p\left|\bar{R}_{t}^{\delta}\right|} \\
& \leq 2 p \sqrt{t \sigma(1)^{2}}+\mathbb{E}^{2 p} \bar{R}_{t}^{\delta}+\mathbb{E}^{-2 p \bar{R}_{t}^{\delta}}
\end{aligned}
$$

It thus suffices to show that $\sup _{0<\delta \leq 1} \mathbb{E} \mathrm{e}^{\beta \bar{R}_{t}^{\delta}}<\infty$ for any $\beta \in \mathbb{R}$. Indeed, we have

$$
\mathbb{E} \mathrm{e}^{\beta \bar{R}_{t}^{\delta}}=\exp \left\{t \int_{\delta<|x| \leq 1}\left(\mathrm{e}^{\beta x}-1-\beta x\right) v(\mathrm{~d} x)\right\}
$$


(a moment generating function of a compensated compound Poisson process). By Taylor's theorem, $\mathrm{e}^{\beta x}-1-\beta x=\beta^{2} x^{2} \mathrm{e}^{\beta \xi} / 2$ for any $|x| \leq 1$, where $\xi$ is some number between 0 and $x$. This completes the proof, as it implies that

$$
\mathbb{E} \mathrm{e}^{\beta \bar{R}_{t}^{\delta}} \leq \exp \left\{\frac{\beta^{2} t}{2} \mathrm{e}^{|\beta|} \int_{|x| \leq 1} x^{2} v(\mathrm{~d} x)\right\} .
$$

Proof of Proposition 4. By the mean value theorem, we have

$$
\mathrm{e}^{M_{t}}-\mathrm{e}^{M_{t}^{\varepsilon}}=\left(M_{t}-M_{t}^{\varepsilon}\right) \mathrm{e}^{\bar{M}_{t}^{\varepsilon}},
$$

where $\bar{M}_{t}^{\varepsilon}$ is between $M_{t}$ and $M_{t}^{\varepsilon}$. Let $q$ be defined such that $1 / p+1 / q=1$. Then

$$
\begin{aligned}
\mathbb{E}\left|\mathrm{e}^{M_{t}}-\mathrm{e}^{M_{t}^{\varepsilon}}\right| & \leq \mathbb{E}\left|M_{t}-M_{t}^{\varepsilon}\right| \mathrm{e}^{\bar{M}_{t}^{\varepsilon}} \\
& \leq \mathbb{E} \sup _{0 \leq s \leq t}\left|R_{s}^{\varepsilon}\right| \mathrm{e}^{\bar{M}_{t}^{\varepsilon}} \\
& \leq\left(\mathbb{E}\left(\sup _{0 \leq s \leq t}\left|R_{s}^{\varepsilon}\right|\right)^{q}\right)^{1 / q}\left(\mathbb{E} \mathrm{e}^{p \bar{M}_{t}^{\varepsilon}}\right)^{1 / p} .
\end{aligned}
$$

Hence, using Doob's inequality and then Proposition 1, we obtain

$$
\begin{aligned}
\mathbb{E}\left|\mathrm{e}^{M_{t}}-\mathrm{e}^{M_{t}^{\varepsilon}}\right| & \leq \frac{q}{q-1}\left(\mathbb{E}\left|R_{t}^{\varepsilon}\right|^{q}\right)^{1 / q}\left(\mathbb{E} \mathrm{e}^{p \bar{M}_{t}^{\varepsilon}}\right)^{1 / p} \\
& \leq C_{p, t} \sigma_{0}(\varepsilon)\left(\mathbb{E}\left(\mathrm{e}^{p M_{t}}+\mathrm{e}^{p M_{t}^{\varepsilon}}\right)\right)^{1 / p},
\end{aligned}
$$

where $C_{p, t}$ denotes a constant depending on $p$ and $t$. We conclude the proof by Lemma 1 .

\subsection{Estimates for cumulative distribution functions}

For cumulative distribution functions, bounds are expected to be bigger. However, in some cases we can get similar results as in the Lipschitz case. In the first result below, we assume local boundedness of the probability density function of the Lévy process $X$ and its supremum process $M$ at a fixed time $t$. The regularity of the probability density function of a Lévy process is studied in [3] and [17]. For the supremum process, see [6] and [9].

Proposition 5. Let $X$ be a Lévy process.

1. If $b>0$ then

$$
\sup _{x \in \mathbb{R}}\left|\mathbb{P}\left[X_{t} \geq x\right]-\mathbb{P}\left[X_{t}^{\varepsilon} \geq x\right]\right| \leq \frac{1}{\sqrt{2 \pi} b} \sigma(\varepsilon) .
$$

2. If $X_{t}$ has a locally bounded probability density function and $x \in \mathbb{R}$, then, for any $q \in(0,1)$,

$$
\left|\mathbb{P}\left[X_{t} \geq x\right]-\mathbb{P}\left[X_{t}^{\varepsilon} \geq x\right]\right| \leq C_{x, t, q} \sigma_{0}(\varepsilon)^{1-q},
$$

where, here and below, $C_{x, t, q}$ denotes a positive constant depending on $x, t$, and $q$.

3. If $M_{t}$ has a locally bounded probability density function on $(0,+\infty)$ and $x>0$, then, for any $q \in\left(0, \frac{1}{2}\right)$,

$$
\left|\mathbb{P}\left[M_{t} \geq x\right]-\mathbb{P}\left[M_{t}^{\varepsilon} \geq x\right]\right| \leq C_{x, t, q} \sigma_{0}(\varepsilon)^{1-q} .
$$


Lemma 2. Let $X$ and $Y$ be two random variables. We assume that $X$ has a bounded density in a neighborhood of $x \in \mathbb{R}$, and that there exists $p>0$ such that $\mathbb{E}|X-Y|^{p}$ is finite. Then there exists a constant $K_{x}>0$ such that, for any $\delta>0$,

$$
|\mathbb{P}[X \geq x]-\mathbb{P}[Y \geq x]| \leq K_{x} \delta+\frac{\mathbb{E}|X-Y|^{p}}{\delta^{p}} .
$$

Proof. We have

$$
|\mathbb{P}[X \geq x]-\mathbb{P}[Y \geq x]|=|\mathbb{P}[X \geq x, Y<x]-\mathbb{P}[X<x, Y \geq x]| .
$$

We will study the above terms on the right-hand side of the equality. We have

$$
\begin{aligned}
\mathbb{P}[X \geq x, Y<x]= & \mathbb{P}[x \leq X<x+(X-Y)] \\
= & \mathbb{P}[x \leq X<x+(X-Y),|X-Y| \leq \delta] \\
& +\mathbb{P}[x \leq X<x+(X-Y),|X-Y|>\delta] \\
\leq & \mathbb{P}[x \leq X<x+\delta]+\mathbb{P}[|X-Y|>\delta] .
\end{aligned}
$$

Suppose that $X$ has a bounded density $f$ in the interval $\left[x-\delta_{0}, x+\delta_{0}\right], \delta_{0}>0$ fixed, and let

$$
K_{x}=\max \left\{\sup _{x-\delta_{0} \leq t \leq x+\delta_{0}} f(t), \frac{1}{\delta_{0}}\right\} .
$$

By considering the cases $\delta<\delta_{0}$ and $\delta \geq \delta_{0}$ separately, it is readily checked that

$$
\mathbb{P}[x \leq X<x+\delta] \leq K_{x} \delta
$$

for any $\delta>0$. Thus, using Markov's inequality, we obtain

$$
\mathbb{P}[X \geq x, Y<x] \leq K_{x} \delta+\frac{\mathbb{E}|X-Y|^{p}}{\delta^{p}} .
$$

Similarly, using $\mathbb{P}[x-\delta \leq X<x] \leq K_{x} \delta$, it holds that

$$
\mathbb{P}[X<x, Y \geq x] \leq K_{x} \delta+\frac{\mathbb{E}|X-Y|^{p}}{\delta^{p}} .
$$

Lemma 2 is thus established.

Proof of Proposition 5. We have

$$
\left|\mathbb{P}\left[X_{t} \geq x\right]-\mathbb{P}\left[X_{t}^{\varepsilon} \geq x\right]\right|=\left|\mathbb{P}\left[X_{t} \geq x, X_{t}^{\varepsilon}<x\right]-\mathbb{P}\left[X_{t}<x, X_{t}^{\varepsilon} \geq x\right]\right| .
$$

It holds that

$\mathbb{P}\left[X_{t} \geq x, X_{t}^{\varepsilon}<x\right]=\mathbb{P}\left[x-\left(X_{t}-X_{t}^{\varepsilon}\right) \leq X_{t}^{\varepsilon}<x\right]=\mathbb{P}\left[x-R_{t}^{\varepsilon} \leq b B_{t}+\left(X_{t}^{\varepsilon}-b B_{t}\right)<x\right]$.

Note that $b B_{t}$ is independent of $X_{t}^{\varepsilon}-b B_{t}$ and $R_{t}^{\varepsilon}$, and $1 /(\sqrt{2 \pi t} b)$ is an upper bound of the probability density function of $b B_{t}$. Then, by conditioning on the pair $\left(R_{t}^{\varepsilon}, X_{t}^{\varepsilon}-b B_{t}\right)$, it can be concluded that

$$
\mathbb{P}\left[x-R_{t}^{\varepsilon} \leq b B_{t}+\left(X_{t}^{\varepsilon}-b B_{t}\right)<x\right] \leq \frac{1}{\sqrt{2 \pi t} b} \mathbb{E}\left|R_{t}^{\varepsilon}\right| .
$$


Therefore, using the fact that $\mathbb{E}\left|R_{t}^{\varepsilon}\right| \leq \sigma(\varepsilon) \sqrt{t}$,

$$
\mathbb{P}\left[X_{t} \geq x, X_{t}^{\varepsilon}<x\right] \leq \frac{1}{\sqrt{2 \pi} b} \sigma(\varepsilon) .
$$

Similarly,

$$
\begin{aligned}
\mathbb{P}\left[X_{t}<x, X_{t}^{\varepsilon} \geq x\right] & =\mathbb{P}\left[x \leq X_{t}^{\varepsilon}<x-\left(X_{t}-X_{t}^{\varepsilon}\right)\right] \\
& =\mathbb{P}\left[x \leq b B_{t}+\left(X_{t}^{\varepsilon}-b B_{t}\right)<x-R_{t}^{\varepsilon}\right] \\
& \leq \frac{1}{\sqrt{2 \pi} b} \sigma(\varepsilon) .
\end{aligned}
$$

Hence, part 1 of the proposition follows from (6).

We now prove part 2 of the proposition. Let $p>0$. By Lemma 2 followed by Proposition 1 , there exist positive constants $K_{x, t}$ and $K_{p, t}$ such that

$$
\begin{aligned}
\left|\mathbb{P}\left[X_{t} \geq x\right]-\mathbb{P}\left[X_{t}^{\varepsilon} \geq x\right]\right| & \leq K_{x, t} \delta+\frac{\mathbb{E}\left|X_{t}-X_{t}^{\varepsilon}\right|^{p}}{\delta^{p}} \\
& =K_{x, t} \delta+\frac{\mathbb{E}\left|R_{t}^{\varepsilon}\right|^{p}}{\delta^{p}} \\
& \leq K_{x, t} \delta+K_{p, t} \frac{\sigma_{0}(\varepsilon)^{p}}{\delta^{p}}
\end{aligned}
$$

for any $\delta>0$. Choosing $\delta=\sigma_{0}(\varepsilon)^{p /(p+1)}$ yields

$$
\left|\mathbb{P}\left[X_{t} \geq x\right]-\mathbb{P}\left[X_{t}^{\varepsilon} \geq x\right]\right| \leq 2 \max \left(K_{x, t}, K_{p, t}\right) \sigma_{0}(\varepsilon)^{p /(p+1)},
$$

and so the result follows since $p /(p+1)$ can be chosen arbitrarily in $(0,1)$.

We now prove part 3 of the proposition. Let $p>1$. By Lemma 2, there exists a constant $K_{x, t}^{\prime}>0$ such that

$$
\left|\mathbb{P}\left[M_{t} \geq x\right]-\mathbb{P}\left[M_{t}^{\varepsilon} \geq x\right]\right| \leq K_{x, t}^{\prime} \delta+\frac{\mathbb{E}\left|M_{t}-M_{t}^{\varepsilon}\right|^{p}}{\delta^{p}}
$$

for any $\delta>0$. On the other hand,

$$
\mathbb{E}\left|M_{t}-M_{t}^{\varepsilon}\right|^{p} \leq \mathbb{E}\left(\sup _{0 \leq s \leq t}\left|X_{s}-X_{s}^{\varepsilon}\right|\right)^{p}=\mathbb{E}\left(\sup _{0 \leq s \leq t}\left|R_{s}^{\varepsilon}\right|\right)^{p} .
$$

So, by Doob's inequality we have, using the constant $K_{p, t}$ from part 2 ,

$$
\mathbb{E}\left|M_{t}-M_{t}^{\varepsilon}\right|^{p} \leq\left(\frac{p}{p-1}\right)^{p} \mathbb{E}\left|R_{t}^{\varepsilon}\right|^{p} \leq K_{p, t}\left(\frac{p}{p-1}\right)^{p} \sigma_{0}(\varepsilon)^{p} .
$$

Part 3 of the proposition then follows by choosing $\delta=\sigma_{0}(\varepsilon)^{p /(p+1)}$.

\section{Approximation of the compensated sum of small jumps by a Brownian motion}

In this section we will replace $R^{\varepsilon}$ by a Brownian motion. This method gives better results, subject to a convergence assumption. In fact, Asmussen and Rosinski proved [1, Theorem 2.1] that, if $X$ is a Lévy process then the process $\sigma(\varepsilon)^{-1} R^{\varepsilon}$ converges in distribution to a standard Brownian motion, when $\varepsilon \rightarrow 0$, if and only if, for any $k>0$,

$$
\lim _{\varepsilon \rightarrow 0} \frac{\sigma(k \sigma(\varepsilon) \wedge \varepsilon)}{\sigma(\varepsilon)}=1 \text {. }
$$


Condition (7) is implied by the condition

$$
\lim _{\varepsilon \rightarrow 0} \frac{\sigma(\varepsilon)}{\varepsilon}=+\infty
$$

Conditions (7) and (8) are equivalent if $v$ does not have atoms in some neighborhood of 0 (see [1, Proposition 2.1]).

\subsection{Estimates for smooth functions}

The errors resulting from the Brownian approximation have not been much studied in the literature, at least theoretically. Some results are given in [7] and [8].

Proposition 6. Let $X$ be an infinite activity Lévy process, and let $t>0$.

1. If $f \in C^{1}(\mathbb{R})$ and satisfies $\mathbb{E}\left|f^{\prime}\left(X_{t}^{\varepsilon}\right)\right|<\infty$, and if there exists $\beta>1$ such that $\left(\sup _{\varepsilon \in(0,1]} \mathbb{E}\left|f^{\prime}\left(X_{t}^{\varepsilon}+\theta \sigma(\varepsilon) \hat{W}_{t}\right)-f^{\prime}\left(X_{t}^{\varepsilon}\right)\right|^{\beta}\right)^{1 / \beta}$ and $\left(\sup _{\varepsilon \in(0,1]} \mathbb{E} \mid f^{\prime}\left(X_{t}^{\varepsilon}+\theta R_{t}^{\varepsilon}\right)-\right.$ $\left.\left.f^{\prime}\left(X_{t}^{\varepsilon}\right)\right|^{\beta}\right)^{1 / \beta}$ are finite and integrable with respect to $\theta$ on $[0,1]$, then

$$
\mathbb{E}\left(f\left(X_{t}\right)-f\left(\hat{X}_{t}^{\varepsilon}\right)\right)=o\left(\sigma_{0}(\varepsilon)\right) .
$$

2. If $f \in C^{2}(\mathbb{R})$ and satisfies $\mathbb{E}\left|f^{\prime}\left(X_{t}^{\varepsilon}\right)\right|+\mathbb{E}\left|f^{\prime \prime}\left(X_{t}^{\varepsilon}\right)\right|<\infty$, and if there exists $\beta>1$ such that $\left(\sup _{\varepsilon \in(0,1]} \mathbb{E}\left|f^{\prime \prime}\left(X_{t}^{\varepsilon}+\theta \sigma(\varepsilon) \hat{W}_{t}\right)-f^{\prime \prime}\left(X_{t}^{\varepsilon}\right)\right|^{\beta}\right)^{1 / \beta}$ and $\left(\sup _{\varepsilon \in(0,1]} \mathbb{E} \mid f^{\prime \prime}\left(X_{t}^{\varepsilon}+\right.\right.$ $\left.\left.\theta R_{t}^{\varepsilon}\right)-\left.f^{\prime \prime}\left(X_{t}^{\varepsilon}\right)\right|^{\beta}\right)^{1 / \beta}$ are finite and integrable with respect to $\theta$ on $[0,1]$, then

$$
\mathbb{E}\left(f\left(X_{t}\right)-f\left(\hat{X}_{t}^{\varepsilon}\right)\right)=o\left(\sigma_{0}(\varepsilon)^{2}\right) .
$$

Examples of functions satisfying the above conditions are noted after Proposition 2.

Proof of Proposition 6. We consider only part 2. The proof for part 1 is similar. By Proposition 2 we have

$$
\mathbb{E}\left(f\left(X_{t}\right)-f\left(X_{t}^{\varepsilon}\right)\right)=\frac{\sigma(\varepsilon)^{2} t}{2} \mathbb{E} f^{\prime \prime}\left(X_{t}^{\varepsilon}\right)+o\left(\sigma_{0}(\varepsilon)^{2}\right) .
$$

On the other hand, using the same reasoning as in the proof of Proposition 2 (we will replace $R^{\varepsilon}$ by $\left.\sigma(\varepsilon) \hat{W}\right)$, we obtain

$$
\mathbb{E}\left(f\left(X_{t}^{\varepsilon}+\sigma(\varepsilon) \hat{W}_{t}\right)-f\left(X_{t}^{\varepsilon}\right)\right)=\frac{\sigma(\varepsilon)^{2} t}{2} \mathbb{E} f^{\prime \prime}\left(X_{t}^{\varepsilon}\right)+o\left(\sigma_{0}(\varepsilon)^{2}\right) .
$$

Hence,

$$
\mathbb{E}\left(f\left(X_{t}\right)-f\left(\hat{X}_{t}^{\varepsilon}\right)\right)=o\left(\sigma_{0}(\varepsilon)^{2}\right) .
$$

The combination of Proposition 6.2 of [7] and Spitzer's identity for Lévy processes (see Proposition 1 of [10]) leads to the following result.

Proposition 7. Let $X$ be an integrable infinite activity Lévy process. Then

$$
\left|\mathbb{E} M_{t}-\mathbb{E} \hat{M}_{t}^{\varepsilon}\right| \leq 33 \sigma(\varepsilon) \rho(\varepsilon)\left(1+\log \left(\frac{\sqrt{t}}{2 \rho(\varepsilon)}\right)\right),
$$

where $\rho(\varepsilon)=\sigma(\varepsilon)^{-3} \int_{|x| \leq \varepsilon}|x|^{3} v(\mathrm{~d} x)$. 
Remark 4. Under condition (8), we have $\lim _{\varepsilon \rightarrow 0} \rho(\varepsilon)=0$ and, in turn,

$$
\sigma(\varepsilon) \rho(\varepsilon)\left(1+\log \left(\frac{\sqrt{t}}{2 \rho(\varepsilon)}\right)\right)=o(\sigma(\varepsilon)) .
$$

Proof of Proposition 7. Let $\delta \in(0, t)$. Using Spitzer's identity for Lévy processes, we have

$$
\begin{aligned}
\left|\mathbb{E} M_{t}-\mathbb{E} \hat{M}_{t}^{\varepsilon}\right| & =\left|\int_{0}^{t} \frac{\mathbb{E} X_{s}^{+}}{s} \mathrm{~d} s-\int_{0}^{t} \frac{\mathbb{E}\left(\hat{X}_{s}^{\varepsilon}\right)^{+}}{s} \mathrm{~d} s\right| \\
& \leq \int_{0}^{\delta}\left|\mathbb{E} X_{s}^{+}-\mathbb{E}\left(\hat{X}_{s}^{\varepsilon}\right)^{+}\right| \frac{\mathrm{d} s}{s}+\int_{\delta}^{t}\left|\mathbb{E} X_{s}^{+}-\mathbb{E}\left(\hat{X}_{s}^{\varepsilon}\right)^{+}\right| \frac{\mathrm{d} s}{s}
\end{aligned}
$$

On the one hand,

$$
\begin{aligned}
\left|\mathbb{E} X_{s}^{+}-\mathbb{E}\left(\hat{X}_{s}^{\varepsilon}\right)^{+}\right| & \leq \mathbb{E}\left|\left(X_{s}^{\varepsilon}+R_{s}^{\varepsilon}\right)^{+}-\left(X_{s}^{\varepsilon}+\sigma(\varepsilon) \hat{W}_{s}\right)^{+}\right| \\
& \leq \mathbb{E}\left|R_{s}^{\varepsilon}-\sigma(\varepsilon) \hat{W}_{s}\right| \\
& \leq\left(1+\sqrt{\frac{2}{\pi}}\right) \sqrt{s} \sigma(\varepsilon) .
\end{aligned}
$$

On the other hand, it follows from Proposition 6.2 of [7] that

$$
\left|\mathbb{E} X_{s}^{+}-\mathbb{E}\left(\hat{X}_{s}^{\varepsilon}\right)^{+}\right| \leq A \sigma(\varepsilon) \rho(\varepsilon),
$$

with $A<16.5$ (consider the function $f(x)=x^{+}$). Therefore,

$$
\begin{aligned}
\left|\mathbb{E} M_{t}-\mathbb{E} \hat{M}_{t}^{\varepsilon}\right| & \leq 2\left(1+\sqrt{\frac{2}{\pi}}\right) \sigma(\varepsilon) \sqrt{\delta}+A \sigma(\varepsilon) \rho(\varepsilon) \log \left(\frac{t}{\delta}\right) \\
& \leq 16.5 \sigma(\varepsilon)\left(\sqrt{\delta}+\rho(\varepsilon) \log \left(\frac{t}{\delta}\right)\right) .
\end{aligned}
$$

The last expression is minimal for $\delta=4 \rho(\varepsilon)^{2}$, and so the desired result follows by substitution.

\subsection{Estimates by Skorokhod embedding}

We will use a powerful tool to prove the results of this section. This is the Skorokhod embedding theorem. We will begin by defining some useful notation.

Definition 1. Define

$$
\begin{aligned}
\beta(\varepsilon) & =\frac{\int_{|x| \leq \varepsilon} x^{4} v(\mathrm{~d} x)}{\left(\sigma_{0}(\varepsilon)\right)^{4}} \\
\beta_{p, \theta}^{t}(\varepsilon) & =\beta(\varepsilon)^{p \theta /(p+4 \theta)}\left[\left(\log \left(\frac{t}{\beta(\varepsilon)^{2 \theta /(p+4 \theta)}}+3\right)\right)^{p}+1\right] \\
\beta_{1}^{t}(\varepsilon) & =\beta(\varepsilon)^{1 / 6}\left(\sqrt{\log \left(\frac{t}{\beta(\varepsilon)^{1 / 3}}+3\right)}+1\right) \\
\beta_{2}^{t}(\varepsilon) & =\beta(\varepsilon)^{1 / 4}\left(\log \left(\frac{t}{\beta(\varepsilon)^{1 / 4}}+3\right)+1\right) .
\end{aligned}
$$

Remark 5. Note that, under condition (8), we have $\lim _{\varepsilon \rightarrow 0} \beta(\varepsilon)=0$. 
The proof of Proposition 7 cannot be extended to the Lipschitz functions, because the reformulation of the Spitzer identity for Lévy processes cannot be applied in that case. We have to use another method. Define

$$
V_{j, n}=R_{j t / n}^{\varepsilon}-R_{(j-1) t / n}^{\varepsilon}, \quad j=1, \ldots, n,
$$

so that $R_{k t / n}^{\varepsilon}=\sum_{j=1}^{k} V_{j, n}, k=1, \ldots, n$. The $V_{j, n}$ are independent and identically distributed (i.i.d.) with the same distribution as $R_{t / n}^{\varepsilon}$; hence, $\mathbb{E}\left(V_{j, n}\right)=0$ and $\operatorname{var}\left(V_{j, n}\right)=\sigma(\varepsilon)^{2} t / n$. Thus, by Skorokhod's embedding theorem (see Theorem 1 of [19, p. 163]), there exist positive i.i.d. random variables $\tau_{j}, j=1, \ldots, n$, and a standard Brownian motion, $\hat{B}$, such that the (partial sums) $R_{k t / n}^{\varepsilon}$ and the $\hat{B}_{\tau_{1}+\cdots+\tau_{k}}, k=1, \ldots, n$, have the same joint distributions; moreover, $\mathbb{E}\left(\tau_{1}\right)=\operatorname{var}\left(V_{1, n}\right)$ and

$$
\mathbb{E} \tau_{1}^{2} \leq 4 \mathbb{E} V_{1, n}^{4}
$$

Furthermore, note that the $\sigma(\varepsilon) \hat{W}_{k t / n}$ and $\hat{B}_{\sigma(\varepsilon)^{2} k t / n}, k=1, \ldots, n$, have the same joint distributions. Set

$$
T_{k}=\tau_{1}+\cdots+\tau_{k}, \quad T_{k}^{\varepsilon}=\frac{\sigma(\varepsilon)^{2} k t}{n} .
$$

This setting will be used in all of the subsequent results.

Theorem 2. Let $X$ be an integrable infinite activity Lévy process, and let $f$ be a Lipschitz function. Then

$$
\left|\mathbb{E} f\left(M_{t}\right)-\mathbb{E} f\left(\hat{M}_{t}^{\varepsilon}\right)\right| \leq C_{t} \sigma_{0}(\varepsilon) \beta_{1}^{t}(\varepsilon),
$$

where $C_{t}$ is a positive constant independent of $\varepsilon$.

Proof. Set

$$
\begin{aligned}
I_{f}^{\varepsilon} & =\left|\mathbb{E}\left(f\left(\sup _{0 \leq s \leq t} X_{s}\right)-f\left(\sup _{0 \leq s \leq t}\left(X_{s}^{\varepsilon}+\sigma(\varepsilon) \hat{W}_{s}\right)\right)\right)\right|, \\
I_{f}^{\varepsilon}(n) & =\left|\mathbb{E}\left(f\left(\sup _{0 \leq k \leq n} X_{k t / n}\right)-f\left(\sup _{0 \leq k \leq n}\left(X_{k t / n}^{\varepsilon}+\sigma(\varepsilon) \hat{W}_{k t / n}\right)\right)\right)\right| .
\end{aligned}
$$

Because $f$ is, say, $K$-Lipschitz, we can show that

$$
\left|f\left(\sup _{0 \leq k \leq n} X_{k t / n}\right)-f\left(\sup _{0 \leq k \leq n}\left(X_{k t / n}^{\varepsilon}+\sigma(\varepsilon) \hat{W}_{k t / n}\right)\right)\right| \leq K\left(\sup _{0 \leq s \leq t}\left|R_{s}^{\varepsilon}\right|+\sigma(\varepsilon) \sup _{0 \leq s \leq t}\left|\hat{W}_{s}\right|\right) .
$$

As the right-hand side expression is integrable, by dominated convergence we can deduce that $\lim _{n \rightarrow+\infty} I_{f}^{\varepsilon}(n)=I_{f}^{\varepsilon}$. It holds that

$$
\begin{aligned}
I_{f}^{\varepsilon}(n) & =\left|\mathbb{E}\left(f\left(\sup _{0 \leq k \leq n}\left(X_{k t / n}^{\varepsilon}+\hat{B}_{T_{k}}\right)\right)-f\left(\sup _{0 \leq k \leq n}\left(X_{k t / n}^{\varepsilon}+\hat{B}_{T_{k}^{\varepsilon}}\right)\right)\right)\right| \\
& \leq K \mathbb{E}\left|\sup _{0 \leq k \leq n}\left(X_{k t / n}^{\varepsilon}+\hat{B}_{T_{k}}\right)-\sup _{0 \leq k \leq n}\left(X_{k t / n}^{\varepsilon}+\hat{B}_{T_{k}^{\varepsilon}}\right)\right| \\
& \leq K \mathbb{E} \sup _{1 \leq k \leq n}\left|\hat{B}_{T_{k}}-\hat{B}_{T_{k}^{\varepsilon}}\right| .
\end{aligned}
$$

Part 1 of the following theorem concludes the proof. 
Theorem 3. Let $X$ be an infinite activity Lévy process.

1. It holds that

$$
\limsup _{n \rightarrow+\infty} \mathbb{E} \sup _{1 \leq k \leq n}\left|\hat{B}_{T_{k}}-\hat{B}_{T_{k}^{\varepsilon}}\right| \leq C_{t} \sigma_{0}(\varepsilon) \beta_{1}^{t}(\varepsilon) .
$$

2. It holds that

$$
\limsup _{n \rightarrow+\infty} \mathbb{E} \sup _{1 \leq k \leq n}\left|\hat{B}_{T_{k}}-\hat{B}_{T_{k}^{\varepsilon}}\right|^{2} \leq C_{t} \sigma_{0}(\varepsilon)^{2} \beta_{2}^{t}(\varepsilon) .
$$

3. For any reals $p \geq 1$ and $\theta \in(0,1)$, it holds that

$$
\limsup _{n \rightarrow+\infty} \mathbb{E} \sup _{1 \leq k \leq n}\left|\hat{B}_{T_{k}}-\hat{B}_{T_{k}^{\varepsilon}}\right|^{p} \leq C_{p, \theta, t} \sigma_{0}(\varepsilon)^{p} \beta_{p, \theta}^{t}(\varepsilon) .
$$

In the above, $C_{t}$ and $C_{p, \theta, t}$ are constants independent of $\varepsilon$.

This theorem is the main result of this section.

Lemma 3. Let $X$ be an infinite activity Lévy process. Then, for any $\delta>0$,

$$
\limsup _{n \rightarrow+\infty} \mathbb{P}\left[\sup _{1 \leq k \leq n}\left|T_{k}-T_{k}^{\varepsilon}\right|>\delta\right] \leq \frac{4 t \sigma_{0}(\varepsilon)^{4} \beta(\varepsilon)}{\delta^{2}} .
$$

Proof. As $T_{k}-T_{k}^{\varepsilon}=\sum_{i=1}^{k}\left(\tau_{i}-\mathbb{E}\left(\tau_{i}\right)\right)$, by Kolmogorov's inequality,

$$
\begin{aligned}
\mathbb{P}\left[\sup _{1 \leq k \leq n}\left|T_{k}-T_{k}^{\varepsilon}\right|>\delta\right] & \leq \frac{\operatorname{var}\left(T_{n}-T_{n}^{\varepsilon}\right)}{\delta^{2}} \\
& \leq \frac{n \operatorname{var}\left(\tau_{1}\right)}{\delta^{2}} \\
& \leq \frac{n \mathbb{E} \tau_{1}^{2}}{\delta^{2}} \\
& \leq \frac{4 n \mathbb{E}\left(R_{t / n}^{\varepsilon}\right)^{4}}{\delta^{2}}
\end{aligned}
$$

where the last inequality follows from (9). The proof then follows from Proposition 1.

Proof of Theorem 3. For $\delta>0$, we have

$$
\mathbb{E} \sup _{1 \leq k \leq n}\left|\hat{B}_{T_{k}}-\hat{B}_{T_{k}^{\varepsilon}}\right|=I_{1}+I_{2}
$$

with

$$
\begin{aligned}
& I_{1}=\mathbb{E} \sup _{1 \leq k \leq n}\left|\hat{B}_{T_{k}}-\hat{B}_{T_{k}^{\varepsilon}}\right| \mathbf{1}_{\left\{\sup _{1 \leq k \leq n}\left|T_{k}-T_{k}^{\varepsilon}\right| \leq \delta\right\}}, \\
& I_{2}=\mathbb{E} \sup _{1 \leq k \leq n}\left|\hat{B}_{T_{k}}-\hat{B}_{T_{k}^{\varepsilon}}\right| \mathbf{1}_{\left\{\sup _{1 \leq k \leq n}\left|T_{k}-T_{k}^{\varepsilon}\right|>\delta\right\}} .
\end{aligned}
$$

On $\left\{\sup _{1 \leq k \leq n}\left|T_{k}-T_{k}^{\varepsilon}\right| \leq \delta\right\}$, set, for fixed $k$,

$$
s_{1}=T_{k}^{\varepsilon} \wedge T_{k}, \quad s_{2}=T_{k}^{\varepsilon} \vee T_{k} .
$$


We have $s_{1} \leq s_{2} \leq s_{1}+\delta$. Let $j$ be such that $j \delta \leq s_{1}<(j+1) \delta$. We have $s_{1} \leq s_{2} \leq(j+2) \delta$. If $j \delta \leq s_{1} \leq s_{2} \leq(j+1) \delta$, we have

$$
\left|\hat{B}_{s_{1}}-\hat{B}_{s_{2}}\right| \leq\left|\hat{B}_{s_{1}}-\hat{B}_{j \delta}\right|+\left|\hat{B}_{j \delta}-\hat{B}_{s_{2}}\right| \leq 2 \sup _{0 \leq j \leq\left[\sigma(\varepsilon)^{2} t / \delta\right]+1}\left(\sup _{j \delta \leq u \leq(j+1) \delta}\left|\hat{B}_{u}-\hat{B}_{j \delta}\right|\right) .
$$

If $j \delta \leq s_{1} \leq(j+1) \delta \leq s_{2} \leq(j+2) \delta$, we have

$$
\begin{aligned}
\left|\hat{B}_{s_{1}}-\hat{B}_{s_{2}}\right| & \leq\left|\hat{B}_{s_{1}}-\hat{B}_{j \delta}\right|+\left|\hat{B}_{j \delta}-\hat{B}_{(j+1) \delta}\right|+\left|\hat{B}_{(j+1) \delta}-\hat{B}_{s_{2}}\right| \\
& \leq 3 \sup _{0 \leq j \leq\left[\sigma(\varepsilon)^{2} t / \delta\right]+2}\left(\sup _{j \delta \leq u \leq(j+1) \delta}\left|\hat{B}_{u}-\hat{B}_{j \delta}\right|\right) .
\end{aligned}
$$

Hence,

$$
\begin{aligned}
I_{1} & \leq 3 \mathbb{E} \sup _{0 \leq j \leq\left[\sigma(\varepsilon)^{2} t / \delta\right]+2}\left(\sup _{j \delta \leq u \leq(j+1) \delta}\left|\hat{B}_{u}-\hat{B}_{j \delta}\right|\right) \\
& =3 \mathbb{E} \sup _{1 \leq j \leq\left[\sigma(\varepsilon)^{2} t / \delta\right]+3}\left(\sup _{(j-1) \delta \leq u \leq j \delta}\left|\hat{B}_{u}-\hat{B}_{(j-1) \delta}\right|\right) .
\end{aligned}
$$

The random variables $\left(\sup _{(j-1) \delta \leq u \leq j \delta}\left|\hat{B}_{u}-\hat{B}_{(j-1) \delta}\right|\right)_{1 \leq j \leq\left[\sigma(\varepsilon)^{2} t / \delta\right]+3}$ are i.i.d. with the same distribution as $\sup _{0 \leq u \leq \delta}\left|\hat{B}_{u}\right|$ and, in turn, $\sqrt{\delta} \sup _{0 \leq u \leq 1}\left|\hat{B}_{u}\right|$. Then

$$
I_{1} \leq 3 \sqrt{\delta} \mathbb{E} \sup _{1 \leq j \leq\left[\sigma(\varepsilon)^{2} t / \delta\right]+3} V_{j}
$$

where the $\left(V_{j}\right)_{1 \leq j \leq\left[\sigma(\varepsilon)^{2} t / \delta\right]+3}$ are i.i.d. random variables with the same distribution as $\sup _{0 \leq u \leq 1}\left|\hat{B}_{u}\right|$. On the other hand, we know that if $\left(V_{j}\right)_{1 \leq j \leq m}$ are i.i.d. random variables satisfying $\mathbb{E}^{\alpha V_{1}^{2}}<\infty$, where $\alpha$ is a positive real, then

$$
\mathbb{E} \sup _{1 \leq j \leq m} V_{j} \leq g\left(m \mathbb{E} \mathrm{e}^{\alpha V_{1}^{2}}\right)
$$

where $g: x \in[1,+\infty) \rightarrow \sqrt{\log (x) / \alpha}$. Indeed, since $g$ is concave, we have

$$
\begin{aligned}
\mathbb{E} \sup _{1 \leq j \leq m} V_{j} & =\mathbb{E} \sup _{1 \leq j \leq m} g\left(\mathrm{e}^{\alpha V_{j}^{2}}\right) \\
& =\mathbb{E} g\left(\sup _{1 \leq j \leq m} \mathrm{e}^{\alpha V_{j}^{2}}\right) \quad \text { (because } g \text { is nondecreasing) } \\
& \leq g\left(\mathbb{E} \sup _{1 \leq j \leq m} \mathrm{e}^{\alpha V_{j}^{2}}\right) \quad \text { (by Jensen's inequality) } \\
& \leq g\left(\mathbb{E} \sum_{j=1}^{m} \mathrm{e}^{\alpha V_{j}^{2}}\right) \quad \text { (because } g \text { is nondecreasing) } \\
& =g\left(m \mathbb{E} \mathrm{e}^{\alpha V_{1}^{2}}\right)
\end{aligned}
$$

In our case $V_{1}=\sup _{0 \leq u \leq 1}\left|\hat{B}_{u}\right|$. So

$$
V_{1} \leq \sup _{0 \leq u \leq 1} \hat{B}_{u}+\sup _{0 \leq u \leq 1}\left(-\hat{B}_{u}\right)
$$


For $\alpha \in\left(0, \frac{1}{8}\right)$, we have

$$
\begin{aligned}
\mathbb{E}^{\alpha V_{1}^{2}} & \left.\leq \mathbb{E} \exp \left\{2 \alpha\left(\sup _{0 \leq u \leq 1} \hat{B}_{u}\right)^{2}+\left(\sup _{0 \leq u \leq 1}\left(-\hat{B}_{u}\right)\right)^{2}\right)\right\} \\
& \leq\left(\mathbb{E} \exp \left\{4 \alpha\left(\sup _{0 \leq u \leq 1} \hat{B}_{u}\right)^{2}\right\}\right)^{1 / 2}\left(\mathbb{E} \exp \left\{4 \alpha\left(\sup _{0 \leq u \leq 1}\left(-\hat{B}_{u}\right)\right)^{2}\right\}\right)^{1 / 2} \\
& =\mathbb{E} \exp \left\{4 \alpha\left(\sup _{0 \leq u \leq 1} \hat{B}_{u}\right)^{2}\right\} \\
& =(1-8 \alpha)^{-1 / 2} .
\end{aligned}
$$

The last equality follows from $\left(\sup _{0 \leq u \leq 1} \hat{B}_{u}\right)^{2} \sim \chi_{1}^{2}$ upon using the moment generating function of the $\chi_{1}^{2}$ distribution, given by $(1-2 \beta)^{-1 / 2}$ for $\beta<\frac{1}{2}$.

It follows straightforwardly from the above that, for $\alpha \in\left(0, \frac{1}{8}\right)$,

$$
I_{1} \leq C_{\alpha} \sqrt{\delta} \sqrt{\log \left(\frac{\sigma(\varepsilon)^{2} t}{\delta}+3\right)}
$$

where

$$
C_{\alpha}=3 \sqrt{\frac{1}{\alpha}\left(1-\frac{\log (1-8 \alpha)}{2 \log (3)}\right)}
$$

Let us now consider $I_{2}$. We have

$$
\begin{aligned}
I_{2} & \leq\left(\mathbb{E}\left(\sup _{1 \leq k \leq n}\left|\hat{B}_{T_{k}}-\hat{B}_{T_{k}^{\varepsilon}}\right|\right)^{2}\right)^{1 / 2}\left(\mathbb{P}\left[\sup _{1 \leq k \leq n}\left|T_{k}-T_{k}^{\varepsilon}\right|>\delta\right]\right)^{1 / 2} \\
& \leq\left(\mathbb{E}\left(\sup _{1 \leq k \leq n}\left|\hat{B}_{T_{k}}\right|+\sup _{1 \leq k \leq n}\left|\hat{B}_{T_{k}^{\varepsilon}}\right|\right)^{2}\right)^{1 / 2}\left(\mathbb{P}\left[\sup _{1 \leq k \leq n}\left|T_{k}-T_{k}^{\varepsilon}\right|>\delta\right]\right)^{1 / 2} \\
& \leq\left(\left(\mathbb{E} \sup _{0 \leq s \leq t}\left|R_{s}^{\varepsilon}\right|^{2}\right)^{1 / 2}+\left(\mathbb{E} \sup _{0 \leq s \leq \sigma(\varepsilon)^{2} t}\left|\hat{B}_{s}\right|^{2}\right)^{1 / 2}\right)\left(\mathbb{P}\left[\sup _{1 \leq k \leq n}\left|T_{k}-T_{k}^{\varepsilon}\right|>\delta\right]\right)^{1 / 2} \\
& \leq 2\left(\left(\mathbb{E}\left|R_{t}^{\varepsilon}\right|^{2}\right)^{1 / 2}+\left(\mathbb{E}\left|\hat{B}_{\sigma(\varepsilon)^{2} t}\right|^{2}\right)^{1 / 2}\right)\left(\mathbb{P}\left[\sup _{1 \leq k \leq n}\left|T_{k}-T_{k}^{\varepsilon}\right|>\delta\right]\right)^{1 / 2} \\
& \leq 4 \sqrt{t} \sigma(\varepsilon)\left(\mathbb{P}\left[\sup _{1 \leq k \leq n}\left|T_{k}-T_{k}^{\varepsilon}\right|>\delta\right]\right)^{1 / 2},
\end{aligned}
$$

where the fourth inequality is obtained using Doob's inequality. So, by Lemma 3 we have

$$
\limsup _{n \rightarrow+\infty} I_{2} \leq 4 \sqrt{t} \sigma(\varepsilon)\left(\frac{4 t \sigma_{0}(\varepsilon)^{4} \beta(\varepsilon)}{\delta^{2}}\right)^{1 / 2} \text {. }
$$

Hence,

$$
\limsup _{n \rightarrow+\infty} \mathbb{E} \sup _{1 \leq k \leq n}\left|\hat{B}_{T_{k}}-\hat{B}_{T_{k}^{\varepsilon}}\right| \leq C_{\alpha} \sqrt{\delta \log \left(\frac{\sigma(\varepsilon)^{2} t}{\delta}+3\right)}+\frac{8 t}{\delta} \sigma(\varepsilon) \sigma_{0}(\varepsilon)^{2} \sqrt{\beta(\varepsilon)} .
$$

Part 1 now follows by letting $C_{t}=\max \left(C_{\alpha}, 8 t\right)$ and choosing $\delta=\sigma_{0}(\varepsilon)^{2} \beta(\varepsilon)^{1 / 3}$.

For the proofs of parts 2 and 3 of the theorem, we refer the reader to [9, pp. 86-89]. However, some small corrections are needed in the proof of part 3 in order to comply with the definition of $\beta_{p, \theta}^{t}(\varepsilon)$. 
Remark 6. Letting $\theta=\frac{1}{2}$ and $p=1,2$ in the definition of $\beta_{p, \theta}^{t}(\varepsilon)$, we see that part 3 of Theorem 3 partially generalizes parts 1 and 2 . It may be relevant to note here that, for part 3 , the proof used the function $g(x)=\left(\alpha^{-1} \log (x)\right)^{p}$, whereas, for parts 1 and 2 , it used the function $g(x)=\left(\alpha^{-1} \log (x)\right)^{p / 2}, p=1,2$, respectively.

The following result follows directly from part 1 of Theorem 3.

Proposition 8. Let $X$ be an integrable infinite activity Lévy process, and let $f$ be a Lipschitz function. Then

$$
\left|\mathbb{E} f\left(X_{t}\right)-\mathbb{E} f\left(\hat{X}_{t}^{\varepsilon}\right)\right| \leq C_{t} \beta_{1}^{t}(\varepsilon) \sigma_{0}(\varepsilon),
$$

where $C_{t}$ is a positive constant.

Proof. We have $R_{t}^{\varepsilon} \stackrel{\mathrm{D}}{=} \hat{B}_{T_{n}}$ and $\sigma(\varepsilon) \hat{W}_{t} \stackrel{\mathrm{D}}{=} \hat{B}_{T_{n}^{\varepsilon}}$. So, if $f$ is $K$-Lipschitz, we have

$$
\left|\mathbb{E} f\left(X_{t}\right)-\mathbb{E} f\left(\hat{X}_{t}^{\varepsilon}\right)\right|=\left|\mathbb{E} f\left(X_{t}^{\varepsilon}+\hat{B}_{T_{n}}\right)-\mathbb{E} f\left(X_{t}^{\varepsilon}+\hat{B}_{T_{n}^{\varepsilon}}\right)\right| \leq K \mathbb{E}\left|\hat{B}_{T_{n}}-\hat{B}_{T_{n}^{\varepsilon}}\right| .
$$

We conclude with Theorem 3 .

For non-Lipschitz functions, we have the following result (corresponding to the payoff of a lookback option).

Proposition 9. Let $X$ be an infinite activity Lévy process, and let $p>1$. If $\mathbb{E}^{p M_{t}}<\infty$ then, for any $x \in \mathbb{R}$ and any $\theta \in(0,1)$,

$$
\left|\mathbb{E}\left(\mathrm{e}^{M_{t}}-x\right)^{+}-\mathbb{E}\left(\mathrm{e}^{\hat{M}_{t}^{\varepsilon}}-x\right)^{+}\right| \leq C_{p, \theta, t} \sigma_{0}(\varepsilon)\left(\beta_{p /(p-1), \theta}^{t}(\varepsilon)\right)^{1-1 / p},
$$

where $C_{p, \theta, t}$ is a positive constant independent of $\varepsilon$.

Proof. Define

$$
M_{t}^{n}=\sup _{0 \leq k \leq n}\left(X_{k t / n}^{\varepsilon}+R_{k t / n}^{\varepsilon}\right), \quad \hat{M}_{t}^{\varepsilon, n}=\sup _{0 \leq k \leq n}\left(X_{k t / n}^{\varepsilon}+\sigma(\varepsilon) \hat{W}_{k t / n}\right) .
$$

We know that $\lim _{n \rightarrow+\infty} M_{t}^{n}=M_{t}$ a.s. and $\lim _{n \rightarrow+\infty} \hat{M}_{t}^{\varepsilon, n}=\hat{M}_{t}^{\varepsilon}$ a.s. Set

$$
U_{t}^{n}=\sup _{0 \leq k \leq n}\left(X_{k t / n}^{\varepsilon}+\hat{B}_{T_{k}}\right), \quad \hat{U}_{t}^{\varepsilon, n}=\sup _{0 \leq k \leq n}\left(X_{k t / n}^{\varepsilon}+\hat{B}_{T_{k}^{\varepsilon}}\right) .
$$

So $M_{t}^{n} \stackrel{\mathrm{D}}{=} U_{t}^{n}$ and $\hat{M}_{t}^{\varepsilon, n} \stackrel{\mathrm{D}}{=} \hat{U}_{t}^{\varepsilon, n}$. By the mean value theorem we have

$$
\mathrm{e}^{U_{t}^{n}}-\mathrm{e}^{\hat{U}_{t}^{\varepsilon, n}}=\left(U_{t}^{n}-\hat{U}_{t}^{\varepsilon, n}\right) \mathrm{e}^{\bar{U}_{t}^{\varepsilon, n}},
$$

where $\bar{U}_{t}^{\varepsilon, n}$ is between $U_{t}^{n}$ and $\hat{U}_{t}^{\varepsilon, n}$. Set

$$
I_{n}^{\varepsilon}=\left|\mathbb{E}\left(\mathrm{e}^{U_{t}^{n}}-x\right)^{+}-\mathbb{E}\left(\mathrm{e}^{\hat{U}_{t}^{\varepsilon, n}}-x\right)^{+}\right| .
$$


Thus,

$$
\begin{aligned}
I_{n}^{\varepsilon} & \leq \mathbb{E}\left|\mathrm{e}^{U_{t}^{n}}-\mathrm{e}^{\hat{U}_{t}^{\varepsilon, n}}\right| \\
& \leq \mathbb{E}\left|U_{t}^{n}-\hat{U}_{t}^{\varepsilon, n}\right| \mathrm{e}^{\bar{U}_{t}^{\varepsilon, n}} \\
& \leq \mathbb{E} \sup _{0 \leq k \leq n}\left|\hat{B}_{T_{k}}-\hat{B}_{T_{k}^{\varepsilon}}\right| \mathrm{e}^{\bar{U}_{t}^{\varepsilon, n}} \\
& \leq\left(\mathbb{E} \sup _{0 \leq k \leq n}\left|\hat{B}_{T_{k}}-\hat{B}_{T_{k}^{\varepsilon}}\right|^{p /(p-1)}\right)^{1-1 / p}\left(\mathbb{E} \mathrm{e}^{p \bar{U}_{t}^{\varepsilon, n}}\right)^{1 / p} \\
& \leq\left(\mathbb{E} \sup _{0 \leq k \leq n}\left|\hat{B}_{T_{k}}-\hat{B}_{T_{k}^{\varepsilon}}\right|^{p /(p-1)}\right)^{1-1 / p}\left(\mathbb{E}\left(\mathrm{e}^{p M_{t}^{n}}+\mathrm{e}^{p \hat{M}_{t}^{\varepsilon, n}}\right)\right)^{1 / p} \\
& \leq\left(\mathbb{E} \sup _{0 \leq k \leq n}\left|\hat{B}_{T_{k}}-\hat{B}_{T_{k}^{\varepsilon}}\right|^{p /(p-1)}\right)^{1-1 / p}\left(\mathbb{E}\left(\mathrm{e}^{p M_{t}}+\mathrm{e}^{p \hat{M}_{t}^{\varepsilon}}\right)\right)^{1 / p} .
\end{aligned}
$$

But,

$$
\begin{aligned}
\mathbb{E}\left(\mathrm{e}^{p M_{t}}+\mathrm{e}^{p \hat{M}_{t}^{\varepsilon}}\right) & \leq \mathbb{E}\left(\mathrm{e}^{p M_{t}}+\exp \left\{p \sigma(\varepsilon) \sup _{0 \leq s \leq t} \hat{W}_{s}\right\} \mathrm{e}^{p M_{t}^{\varepsilon}}\right) \\
& \leq \mathbb{E} \mathrm{e}^{p M_{t}}+2 \mathrm{e}^{p^{2} \sigma(\varepsilon)^{2} t / 2} \mathbb{E} \mathrm{e}^{p M_{t}^{\varepsilon}} \\
& \leq 2 \mathrm{e}^{p^{2} \sigma(\varepsilon)^{2} t / 2} \mathbb{E}\left(\mathrm{e}^{p M_{t}}+\mathrm{e}^{p M_{t}^{\varepsilon}}\right) .
\end{aligned}
$$

So, using dominated convergence, Theorem 3, and Lemma 1, we obtain

$$
\begin{aligned}
\left|\mathbb{E}\left(\mathrm{e}^{M_{t}}-x\right)^{+}-\mathbb{E}\left(\mathrm{e}^{\hat{M}_{t}^{\varepsilon}}-x\right)^{+}\right| & =\lim _{n \rightarrow+\infty}\left|\mathbb{E}\left(\mathrm{e}^{M_{t}^{n}}-x\right)^{+}-\mathbb{E}\left(\mathrm{e}^{\hat{M}_{t}^{\varepsilon, n}}-x\right)^{+}\right| \\
& =\limsup _{n \rightarrow+\infty}\left|\mathbb{E}\left(\mathrm{e}^{U_{t}^{n}}-x\right)^{+}-\mathbb{E}\left(\mathrm{e}^{\hat{U}_{t}^{\varepsilon, n}}-x\right)^{+}\right| \\
& \leq C_{p, \theta, t} \sigma_{0}(\varepsilon)\left(\beta_{p /(p-1), \theta}^{t}(\varepsilon)\right)^{1-1 / p}
\end{aligned}
$$

\subsection{Estimates for cumulative distribution functions}

The bounds obtained in this section are better than those obtained by truncation, provided that condition (8) is satisfied.

Proposition 10. Let $X$ be an infinite activity Lévy process. Below, the constants $C_{t}$ and $C_{x, t, q, \theta}$ are independent of $\varepsilon$.

1. If $b>0$ then

$$
\sup _{x \in \mathbb{R}}\left|\mathbb{P}\left[X_{t} \geq x\right]-\mathbb{P}\left[\hat{X}_{t}^{\varepsilon} \geq x\right]\right| \leq C_{t} \sigma_{0}(\varepsilon) \beta_{1}^{t}(\varepsilon) .
$$

2. If $X_{t}$ has a locally bounded probability density function and $x \in \mathbb{R}$, then, for any pair of reals $\theta \in(0,1), q \in\left(0, \frac{1}{2}\right]$,

$$
\left|\mathbb{P}\left[X_{t} \geq x\right]-\mathbb{P}\left[\hat{X}_{t}^{\varepsilon} \geq x\right]\right| \leq C_{x, t, q, \theta} \sigma_{0}(\varepsilon)^{1-q}\left(\beta_{1 / q-1, \theta}^{t}(\varepsilon)\right)^{q} .
$$

3. If $M_{t}$ has a locally bounded probability density function on $(0,+\infty)$ and $x>0$, then, for any pair of reals $\theta \in(0,1), q \in\left(0, \frac{1}{2}\right]$,

$$
\left|\mathbb{P}\left[M_{t} \geq x\right]-\mathbb{P}\left[\hat{M}_{t}^{\varepsilon} \geq x\right]\right| \leq C_{x, t, q, \theta} \sigma_{0}(\varepsilon)^{1-q}\left(\beta_{1 / q-1, \theta}^{t}(\varepsilon)\right)^{q} .
$$


Proof. Recall that $R_{t}^{\varepsilon} \stackrel{\mathrm{D}}{=} \hat{B}_{T_{n}}$ and $\sigma(\varepsilon) \hat{W}_{t} \stackrel{\mathrm{D}}{=} \hat{B}_{T_{n}^{\varepsilon}}$. Set

$$
Y_{t}=X_{t}^{\varepsilon}+\hat{B}_{T_{n}}, \quad \hat{Y}_{t}^{\varepsilon}=X_{t}^{\varepsilon}+\hat{B}_{T_{n}^{\varepsilon}} .
$$

Thus,

$$
\begin{aligned}
\left|\mathbb{P}\left[X_{t} \geq x\right]-\mathbb{P}\left[\hat{X}_{t}^{\varepsilon} \geq x\right]\right| & =\left|\mathbb{P}\left[Y_{t} \geq x\right]-\mathbb{P}\left[\hat{Y}_{t}^{\varepsilon} \geq x\right]\right| \\
& =\left|\mathbb{P}\left[Y_{t} \geq x, \hat{Y}_{t}^{\varepsilon}<x\right]-\mathbb{P}\left[Y_{t}<x, \hat{Y}_{t}^{\varepsilon} \geq x\right]\right| .
\end{aligned}
$$

It holds that

$$
\begin{aligned}
\mathbb{P}\left[Y_{t} \geq x, \hat{Y}_{t}^{\varepsilon}<x\right] & =\mathbb{P}\left[x-\left(Y_{t}-\hat{Y}_{t}^{\varepsilon}\right) \leq \hat{Y}_{t}^{\varepsilon}<x\right] \\
& =\mathbb{P}\left[x-\left(\hat{B}_{T_{n}}-\hat{B}_{T_{n}^{\varepsilon}}\right) \leq b B_{t}+\left(\hat{Y}_{t}^{\varepsilon}-b B_{t}\right)<x\right] .
\end{aligned}
$$

By construction, $b B_{t}$ is independent of $\left(\hat{Y}_{t}^{\varepsilon}-b B_{t}\right)$ and of $\left(\hat{B}_{T_{n}}-\hat{B}_{T_{n}^{\varepsilon}}\right)$. Furthermore, $1 /(b \sqrt{2 \pi t})$ is an upper bound of the probability density function of $b B_{t}$. By conditioning on the pair $\left(\hat{B}_{T_{n}}-\hat{B}_{T_{n}^{\varepsilon}}, \hat{Y}_{t}^{\varepsilon}-b B_{t}\right)$, it can thus be concluded that

$$
\mathbb{P}\left[Y_{t} \geq x, \hat{Y}_{t}^{\varepsilon}<x\right] \leq \frac{1}{b \sqrt{2 \pi t}} \mathbb{E}\left|\hat{B}_{T_{n}}-\hat{B}_{T_{n}^{\varepsilon}}\right|
$$

Analogously, it also holds that

$$
\mathbb{P}\left[Y_{t}<x, \hat{Y}_{t}^{\varepsilon} \geq x\right] \leq \frac{1}{b \sqrt{2 \pi t}} \mathbb{E}\left|\hat{B}_{T_{n}}-\hat{B}_{T_{n}^{\varepsilon}}\right|
$$

We get the first part of the proposition by using Theorem 3 .

We now prove the second part of the proposition. Let $p \geq 1$. By Lemma 2, there exists $K_{x, t}>0$ such that, for any $\delta>0$,

$$
\left|\mathbb{P}\left[Y_{t} \geq x\right]-\mathbb{P}\left[\hat{Y}_{t}^{\varepsilon} \geq x\right]\right| \leq K_{x, t} \delta+\frac{\mathbb{E}\left|Y_{t}-\hat{Y}_{t}^{\varepsilon}\right|^{p}}{\delta^{p}}=K_{x, t} \delta+\frac{\mathbb{E}\left|\hat{B}_{T_{n}}-\hat{B}_{T_{n}^{\varepsilon}}\right|^{p}}{\delta^{p}} .
$$

Hence, given $\theta \in(0,1)$, by Theorem 3 , there exists a constant $C_{p, \theta, t}>0$ such that

$$
\left|\mathbb{P}\left[Y_{t} \geq x\right]-\mathbb{P}\left[\hat{Y}_{t}^{\varepsilon} \geq x\right]\right| \leq K_{x, t} \delta+C_{p, \theta, t} \frac{\sigma_{0}(\varepsilon)^{p} \beta_{p, \theta}^{t}(\varepsilon)}{\delta^{p}} .
$$

Choosing $\delta=\sigma_{0}(\varepsilon)^{p /(p+1)} \beta_{p, \theta}^{t}(\varepsilon)^{1 /(p+1)}$ yields

$$
\left|\mathbb{P}\left[Y_{t} \geq x\right]-\mathbb{P}\left[\hat{Y}_{t}^{\varepsilon} \geq x\right]\right| \leq 2 \max \left(K_{x, t}, C_{p, \theta, t}\right) \sigma_{0}(\varepsilon)^{p /(p+1)} \beta_{p, \theta}^{t}(\varepsilon)^{1 /(p+1)} .
$$

The result then follows by substituting $p=1 / q-1$.

For the third part of the proposition, we use the notation of Proposition 9. Note that

$$
\begin{aligned}
\left|\mathbb{P}\left[M_{t} \geq x\right]-\mathbb{P}\left[\hat{M}_{t}^{\varepsilon} \geq x\right]\right| & =\lim _{n \rightarrow \infty}\left|\mathbb{P}\left[M_{t}^{n} \geq x\right]-\mathbb{P}\left[\hat{M}_{t}^{\varepsilon, n} \geq x\right]\right| \\
& =\lim _{n \rightarrow \infty}\left|\mathbb{P}\left[U_{t}^{n} \geq x\right]-\mathbb{P}\left[\hat{U}_{t}^{\varepsilon, n} \geq x\right]\right| .
\end{aligned}
$$

Let $p \geq 1$, and put $I_{x, \delta}=[x-\delta, x+\delta)$. Using the proof of Lemma 2, we have, for any $\delta>0$,

$$
\begin{aligned}
\left|\mathbb{P}\left[U_{t}^{n} \geq x\right]-\mathbb{P}\left[\hat{U}_{t}^{\varepsilon, n} \geq x\right]\right| & \leq \mathbb{P}\left[U_{t}^{n} \in I_{x, \delta}\right]+\frac{\mathbb{E}\left|U_{t}^{n}-\hat{U}_{t}^{\varepsilon, n}\right|^{p}}{\delta^{p}} \\
& \leq \mathbb{P}\left[M_{t}^{n} \in I_{x, \delta}\right]+\frac{\mathbb{E} \sup _{1 \leq k \leq n}\left|\hat{B}_{T_{k}}-\hat{B}_{T_{k}^{\varepsilon}}\right|^{p}}{\delta^{p}} .
\end{aligned}
$$


By the assumption on $M_{t}$, there exists a constant $K_{x, t}^{\prime}>0$ such that $\mathbb{P}\left[M_{t} \in I_{x, \delta}\right]<K_{x, t}^{\prime} \delta$ for any $\delta>0$. Combined with Theorem 3 , letting $n \rightarrow \infty$ yields

$$
\lim _{n \rightarrow \infty}\left|\mathbb{P}\left[U_{t}^{n} \geq x\right]-\mathbb{P}\left[\hat{U}_{t}^{\varepsilon, n} \geq x\right]\right| \leq K_{x, t}^{\prime} \delta+C_{p, \theta, t} \frac{\sigma_{0}(\varepsilon)^{p} \beta_{p, \theta}^{t}(\varepsilon)}{\delta^{p}}
$$

for some constant $C_{p, \theta, t}>0$. So, as in part 2 , the result follows by choosing $\delta=\sigma_{0}(\varepsilon)^{p /(p+1)}$ $\beta_{p, \theta}^{t}(\varepsilon)^{1 /(p+1)}$.

\section{References}

[1] Asmussen, S. And Rosinski, J. (2001). Approximations of small jumps of Lévy processes with a view towards simulation. J. Appl. Prob. 38, 482-493.

[2] BarndorfF-Nielsen, O. E. (1997). Normal inverse Gaussian distributions and stochastic volatility modelling. Scand. J. Statist. 24, 1-13.

[3] Bertoin, J. (1996). Lévy Processes. Cambridge University Press.

[4] Broadie, M. And Yamamoto, Y. (2005). A double-exponential fast Gauss transform algorithm for pricing discrete path-dependent options. Operat. Res. 53, 764-779.

[5] Carr, P., Geman, H., Madan, D. B. And Yor, M. (2002). The fine structure of asset returns: an empirical investigation. J. Business. 75, 305-332.

[6] Chaumont, L. (2013). On the law of the supremum of Lévy processes. To appear in Ann. Prob.

[7] Cont, R. And Tankov, P. (2004). Financial Modelling with Jump Processes. Chapman \& Hall, Boca Raton, FL.

[8] Cont, R. And Voltchkova, E. (2005). Integro-differential equations for option prices in exponential Lévy models. Finance Stoch. 9, 299-325.

[9] DiA, E. H. A. (2010). Options exotiques dans les modèles exponentiels de Lévy. Doctoral Thesis, Université Paris-Est. Available at http://tel.archives-ouvertes.fr/tel-00520583/en/.

[10] Dia, E. H. A. And Lamberton, D. (2011). Connecting discrete and continuous lookback or hindsight options in exponential Lévy models. Adv. Appl. Prob. 43, 1136-1165.

[11] Eberlein, E. (2001). Application of generalized hyperbolic Lévy motions to finance. In Lévy Processes, eds O. E. Barndorff-Nielsen, T. Mikosch and S. Resnick. Birkhäuser, Boston, MA, pp. 319-337.

[12] Feng, L. And Linetsky, V. (2008). Pricing discretely monitored barrier options and defaultable bonds in Lévy process models: a fast Hilbert transform approach. Math. Finance 18, 337-384.

[13] Feng, L. And Linetsky, V. (2009). Computing exponential moments of the discrete maximum of a Lévy process and lookback options. Finance Stoch. 13, 501-529.

[14] Morris, C. N. (1982). Natural exponential families with quadratic variance functions. Ann. Statist. 10, 65-80.

[15] Petrella, G. And Kou, S. G. (2004). Numerical pricing of discrete barrier and lookback options via Laplace transforms. J. Comput. Finance 8, 1-37.

[16] Rydberg, T. H. (1997). The normal inverse Gaussian Lévy process: simulation and approximation. Commun. Statist. Stoch. Models 13, 887-910.

[17] Sato, K.-I. (1999). Lévy Processes and Infinitely Divisible Distributions. Cambridge University Press.

[18] SignahL, M. (2003). On error rates in normal approximations and simulation schemes for Lévy processes. Stoch. Models 19, 287-298.

[19] Sкоrokнod, A. V. (1965). Studies in the Theory of Random Processes. Addison-Wesley, Reading, MA.

[20] TANkov, P. (2004). Lévy processes in finance: inverse problems and dependence modelling. Doctoral Thesis, Ecole Polytechnique. 